TRANSACTIONS OF THE

AMERICAN MATHEMATICAL SOCIETY

Volume 360, Number 5, May 2008, Pages 2741-2765

S 0002-9947(07)04440-6

Article electronically published on December 20, 2007

\title{
PSEUDOCYCLES AND INTEGRAL HOMOLOGY
}

\author{
ALEKSEY ZINGER
}

\begin{abstract}
We describe a natural isomorphism between the set of equivalence classes of pseudocycles and the integral homology groups of a smooth manifold. Our arguments generalize to settings well-suited for applications in enumerative algebraic geometry and for construction of the virtual fundamental class in the Gromov-Witten theory.
\end{abstract}

\section{INTRODUCTION}

1.1. Main theorem. In his seminal paper $[G]$, Gromov initiated the study of pseudoholomorphic curves in symplectic manifolds and demonstrated their usefulness by proving a number of important results in symplectic topology. In [McSa] and [RT], pseudoholomorphic curves are used to define invariants of semipositive manifolds. In particular, it is shown in $[\mathrm{McSa}]$ and $[\mathrm{RT}]$ that for every compact semipositive symplectic manifold $(X, \omega)$, homology class $A \in H_{2}(X ; \mathbb{Z})$, integers $k \geq 3$ and $N \geq 0$, and generic compatible almost complex structure $J$ on $X$, there exists a smooth oriented manifold $\mathcal{M}_{k, N}(A, J)$ and a smooth map

$$
\mathrm{ev}_{k, N}^{A, J}: \mathcal{M}_{k, N}(A, J) \longrightarrow X^{k+N}
$$

such that the "boundary" of $\operatorname{ev}_{k, N}^{A, J}$ is small; see below. Such a smooth map is called a pseudocycle and determines a homomorphism $H_{*}\left(X^{k+N} ; \mathbb{Z}\right) \longrightarrow \mathbb{Z}$, which turns out to be an invariant of $(X, \omega ; A, k, N)$.

In general, if $X$ is a smooth manifold, subset $Z$ of $X$ is said to have dimension at most $k$ if there exists a $k$-dimensional manifold $Y$ and a smooth map $h: Y \longrightarrow X$ such that the image of $h$ contains $Z$. If $f: M \longrightarrow X$ is a continuous map between topological spaces, the boundary of $f$ is the set

$$
\operatorname{Bd} f=\bigcap_{K \subset M \text { cmpt }} \overline{f(M-K)} \text {. }
$$

A smooth map $f: M \longrightarrow X$ is a $k$-pseudocycle if $M$ is an oriented $k$-manifold, $f(M)$ is a pre-compact ${ }^{1}$ subset of $X$, and the dimension of $\mathrm{Bd} f$ is at most $k-2$. Two $k$-pseudocycles $f_{0}: M_{0} \longrightarrow X$ and $f_{1}: M_{1} \longrightarrow X$ are equivalent if there exists a smooth oriented manifold $\tilde{M}$ and a smooth map $\tilde{f}: \tilde{M} \longrightarrow X$ such that the image of $\tilde{f}$ is a pre-compact subset of $X$,

$$
\operatorname{dim} \operatorname{Bd} \tilde{f} \leq k-1, \quad \partial \tilde{M}=M_{1}-M_{0},\left.\quad \tilde{f}\right|_{M_{0}}=f_{0}, \quad \text { and }\left.\quad \tilde{f}\right|_{M_{1}}=f_{1} .
$$

Received by the editors May 19, 2006 and, in revised form, October 5, 2006.

2000 Mathematics Subject Classification. Primary 55N99, 57R95.

Key words and phrases. Pseudocycles, homology, symplectic geometry.

${ }^{1}$ I.e. its closure is compact. 
We denote the set of equivalence classes of pseudocycles into $X$ by $\mathcal{H}_{*}(X)$. This set is naturally a $\mathbb{Z}$-graded module over $\mathbb{Z}$. In this paper, we prove

Theorem 1.1. If $X$ is a smooth manifold, there exist natural ${ }^{2}$ homomorphisms of graded $\mathbb{Z}$-modules

$$
\Psi_{*}: H_{*}(X ; \mathbb{Z}) \longrightarrow \mathcal{H}_{*}(X) \text { and } \Phi_{*}: \mathcal{H}_{*}(X) \longrightarrow H_{*}(X ; \mathbb{Z}),
$$

such that $\Phi_{*} \circ \Psi_{*}=\mathrm{Id}$ and $\Psi_{*} \circ \Phi_{*}=\mathrm{Id}$.

Remark 1. In [McSa] and [RT], a pseudocycle is not explicitly required to have a pre-compact image. As $[\mathrm{McSa}]$ and $[\mathrm{RT}]$ work with compact manifolds, this condition is automatically satisfied. However, this requirement is essential in the noncompact case. As observed in $[\mathrm{K}]$, there is no surjective homomorphism from $H_{*}(X ; \mathbb{Z})$ to $\mathcal{H}_{*}(X)$ if $X$ is not compact and pseudocycles are not required to have pre-compact images.

Remark 2. It is sufficient to require that a pseudocycle map be continuous, as long as the same condition is imposed on pseudocycle equivalences. All arguments in this paper go through for continuous pseudocycles. In fact, Lemma 2.1 is no longer necessary. However, smooth pseudocycles are useful in symplectic topology for defining invariants as intersection numbers.

In order to define symplectic invariants, $[\mathrm{McSa}]$ and $[\mathrm{RT}]$ observe that every element of $\mathcal{H}_{*}(X)$ defines a homomorphism $H_{*}(X ; \mathbb{Z}) \longrightarrow \mathbb{Z}$, or equivalently an element of $H_{*}(X ; \mathbb{Z}) / \operatorname{Tor}\left(H_{*}(X ; \mathbb{Z})\right)$. Thus, Theorem 1.1 leads to symplectic invariants that may be strictly stronger than the $\mathrm{GW}$-invariants defined in [McSa] and in [RT]. In fact, they are as good as the maps $\operatorname{ev}_{k, N}^{A, J}$ can give:

Corollary 1.2. If $\left(X, \omega_{1}\right)$ and $\left(X, \omega_{2}\right)$ are semipositive symplectic manifolds that have the same $G W$-invariants, viewed as a collection of integral homology classes, then the corresponding collections of evaluation maps from products of moduli spaces of pseudoholomorphic maps and Riemannian surfaces are equivalent as pseudocycles.

This corollary is immediate from Theorem 1.1.

This paper was begun while the author was a graduate student at MIT and then put on the back burner. Its aim was to clarify relations between $H_{*}(X ; \mathbb{Z})$ and $\mathcal{H}_{*}(X)$ that were hinted at in [McSa] and stated without a proof in [RT]. Since then, this issue has been explored in $[\mathrm{K}]$ and in $[\mathrm{Sc}]$. The views taken in $[\mathrm{K}]$ and in $[\mathrm{Sc}]$ differ significantly from the present paper. In particular, while non-compact manifolds are considered in $[\mathrm{K}]$, pseudocycles in $[\mathrm{K}]$ are not required to have precompact images. Theorem 1.1 fails for such pseudocycles. The arguments in the present paper are rather direct and use no advanced techniques, beyond standard algebraic topology. In a sense they implement an outline proposed in Section 7.1 of $[\mathrm{McSa}]$. However, a fully rigorous implementation of this outline requires nontrivial technical facts obtained in Subsections 2.2 and 2.3 of this paper. An additional obstacle arises in showing that the map $\Psi_{*}$ is well-defined. As discussed in the next subsection, there are two ways of overcoming it.

As a graduate student, the author was partially supported by an NSF Graduate Research Fellowship and NSF grant DMS-9803166. The author would like to thank

\footnotetext{
${ }^{2}$ In other words, $\Psi_{*}$ and $\Phi_{*}$ are natural transformations of functors $H_{*}(\cdot ; \mathbb{Z})$ and $\mathcal{H}_{*}(\cdot)$ from the category of smooth compact manifolds and maps.
} 
D. McDuff for a suggestion that greatly simplified one of the steps in the original argument and for comments on an earlier draft of the present version that led to improvements in the exposition.

1.2. Outline of constructions. If $M$ is a compact oriented $k$-manifold and $f$ : $M \longrightarrow X$ is a smooth map, $f$ determines an element of $H_{k}(X ; \mathbb{Z})$, i.e. the pushforward of the fundamental class of $M, f_{*}[M]$. If $f: M \longrightarrow X$ is a $k$-pseudocycle, $M$ need not be compact. However, one can choose a compact $k$-submanifold with boundary, $\bar{V} \subset M$, so that $f(M-V)$ lies in a small neighborhood $U$ of $\operatorname{Bd} f$. In particular, $\left.f\right|_{\bar{V}}$ determines the homology class

$$
f_{*}[\bar{V}] \in H_{k}(X, U ; \mathbb{Z}) .
$$

By Proposition $2.2, U$ can be chosen so that $H_{k}(X, U ; \mathbb{Z})$ is naturally isomorphic to $H_{k}(X ; \mathbb{Z})$. In order to show that the image of $f_{*}[V]$ in $H_{k}(X ; \mathbb{Z})$ depends only on $f$ (and not $V$ or $U$ ), we use Proposition 2.9 to replace the singular chain complex $S_{*}(X)$ by a quotient complex $\bar{S}_{*}(X)$. The advantage of the latter complex is that cycles and boundaries between chains can be constructed more easily.

In an analogous way, a pseudocycle equivalence $\tilde{f}: \tilde{M} \longrightarrow X$ between two pseudocycles

$$
f_{i}: M_{i} \longrightarrow X, \quad i=0,1,
$$

gives rise to a chain equivalence between the corresponding cycles in $\bar{S}_{*}(X, W)$, for a small neighborhood $W$ of $\mathrm{Bd} \tilde{f}$. In particular, the homology cycles determined by $f_{0}$ and $f_{1}$ are equal in $H_{k}(X, W ; \mathbb{Z})$. On the other hand, by Proposition 2.2 , $W$ can be chosen so that $H_{k}(X ; \mathbb{Z})$ naturally injects into $H_{k}(X, W ; \mathbb{Z})$. Therefore, $f_{0}$ and $f_{1}$ determine the same elements of $H_{k}(X ; \mathbb{Z})$, and the homomorphism $\Phi_{*}$ is well-defined. Its construction is described in detail in Subsection 3.2.

Remark 1. The homomorphism

$$
\Phi_{*}: \mathcal{H}_{*}(X) \longrightarrow H_{*}(X ; \mathbb{Z})
$$

of Subsection 3.2 induces the linear map

$$
\mathcal{H}_{*}(X) \longrightarrow H_{*}(X ; \mathbb{Z}) / \operatorname{Tor}\left(H_{*}(X ; \mathbb{Z})\right)
$$

described in $[\mathrm{McSa}]$ and $[\mathrm{RT}]$. However, our construction of $\Phi_{*}$ differs from that of the induced map in $[\mathrm{McSa}]$ and $[\mathrm{RT}]$. Indeed, the latter is constructed via the homomorphism $\Psi_{*}$ and a natural intersection pairing on $\mathcal{H}_{*}(X)$. The construction of $\Phi_{*}$ in Subsection 3.2 is more direct.

Remark 2. The construction of Subsection 3.2 implies the following. Suppose $M$ is an oriented $k$-manifold and $f: M \longrightarrow X$ is a continuous map with a pre-compact image. Suppose $\operatorname{Bd} f$ has an arbitrary small neighborhood $U$ so that

$$
H_{l}(U)=0 \quad \forall l>k-2 .
$$

Then, $f$ defines an element in $H_{k}(X) .^{3}$ An analogous statement holds for equivalences between maps. Note that we are not assuming that $X$ is a smooth manifold. These observations have a variety of applications. For example, the first statement implies that a compact complex algebraic variety carries a fundamental class. For essentially the same reason, (generalized) pseudocycles figure prominently in the approach in [Z1] to a large class of problems in enumerative geometry. Pseudocycles

\footnotetext{
${ }^{3}$ This statement holds for any coefficient ring.
} 
can also be used to give a more geometric interpretation of the virtual fundamental class construction of [FO] and [LT] and are used to define new symplectic invariants in [Z2]. This is a different type of generalization, as the ambient space $X$ in these settings is a topological space stratified by infinite-dimensional manifolds.

In order to construct the homomorphism $\Psi_{*}$, we would like to show that a singular cycle gives rise to a pseudocycle and a chain equivalence between two cycles gives rise to a pseudocycle equivalence between the corresponding pseudocycles. The former works out precisely as outlined in Section 7.1 of [McSa], with a reinterpretation for the complex $\bar{S}_{*}(X){ }^{4}$ If $s$ is a $k$-cycle, all codimension-one simplices of its $k$ simplices must cancel in pairs. By gluing the $k$-simplices along the codimension-one faces paired in this way, we obtain a continuous map from a compact topological space $M^{\prime}$ to $X$. The complement of the codimension-two simplices is a smooth manifold and the continuous map can be smoothed out in a fixed manner using Lemma 2.1. We thus obtain a pseudocycle from the cycle $s$.

On the other hand, turning a chain equivalence $\tilde{s}$ between two $k$-cycles, $s_{0}$ and $s_{1}$, into a pseudocycle equivalence between the corresponding pseudocycles,

$$
f_{0}: M_{0} \longrightarrow X \quad \text { and } \quad f_{1}: M_{1} \longrightarrow X,
$$

turns out to be less straightforward. Similarly to the previous paragraph, $\tilde{s}$ gives rise to a smooth map from a smooth $(k+1)$-manifold with boundary,

$$
\tilde{f}: \tilde{M}^{*} \longrightarrow X .
$$

However, if all codimension-two simplices (including those of dimension $k-1$ ) are dropped, the boundary of $\tilde{M}^{*}$ will be the complement in $M_{0} \sqcup M_{1}$ of a subset of dimension $k-1$ (instead of being $M_{0} \sqcup M_{1}$ ). One way to fix this is to keep the $(k-1)$-simplices that would lie on the boundary. In such a case, the entire space may no longer be a smooth manifold and its boundary may not be $M_{0} \sqcup M_{1}$, because the $(k-1)$-simplices of the $(k+1)$-simplices of $\tilde{s}$ may be identified differently from the way the $(k-1)$-simplices of the $k$-simplices of $s_{0}$ and $s_{1}$ are identified. It is possible to modify $\tilde{s}$ so that all identifications are consistent. However, the required modification turns out to be quite laborious. This direct approach was implemented in the original version of this paper (now called Version B).

In the present version, we instead implement a less direct, but far simpler, construction which is based on a suggestion of D. McDuff. Instead of trying to reinsert $(k-1)$-simplices into the boundary of $\tilde{M}^{*}$, we will attach to $\tilde{M}^{*}$ two collars,

$$
\tilde{M}_{0} \subset[0,1] \times M_{0} \quad \text { and } \quad \tilde{M}_{1} \subset[0,1] \times M_{1} .
$$

The boundary of $\tilde{M}_{i}$ will have two pieces, $M_{i}$ and the complement in $M_{i}$ of the $(k-1)$ simplices. We attach the latter to the piece of the boundary of $\tilde{M}^{*}$ corresponding to $s_{i}$. In this way, we obtain a smooth manifold $\tilde{M}$ with boundary $M_{1}-M_{0}$ and a pseudocycle equivalence from $f_{0}$ to $f_{1}$; see Subsection 3.1 for details.

Finally, in Subsection 3.3, we verify that the homomorphisms $\Psi_{*}$ and $\Phi_{*}$ are mutual inverses. It is fairly straightforward to see that the map $\Phi_{*} \circ \Psi_{*}$ is the identity on $\bar{H}_{*}(X ; \mathbb{Z})$. However, showing the injectivity of $\Phi_{*}$ requires more care. The desired pseudocycle equivalence,

$$
\tilde{f}: \tilde{M} \longrightarrow X,
$$

\footnotetext{
${ }^{4}$ This reinterpretation is not necessary to construct the map $\Psi_{*}$ in Subsection 3.1 However, it is needed in Subsection 3.3 to show that the maps $\Phi_{*}$ and $\Psi_{*}$ are isomorphisms.
} 
is constructed by taking a limit of the corresponding construction in Subsection 3.1. In particular, the smooth manifold $\tilde{M}$ is obtained as a subspace of a non-compact space.

\section{Preliminaries}

2.1. Notation. In this subsection we describe a number of subsets of the standard $k$-simplex $\Delta^{k}$ as well as maps between standard simplices of various dimensions. We conclude by constructing self-maps of $\Delta^{k}$ which will be used to define canonical smoothings of piecewise smooth maps in Subsection 3.1; see Lemma 2.1. The reader may want to skip this subsection at first and refer back for notation as needed.

If $A$ is a finite subset of $\mathbb{R}^{k}$, we denote by $\mathrm{CH}(A)$ and $\mathrm{CH}^{0}(A)$ the (closed) convex hull of $A$ and the open convex hull of $A$, respectively, i.e.

$$
\begin{aligned}
C H(A) & =\left\{\sum_{v \in A} t_{v} v: t_{v} \in[0,1] ; \sum_{v \in A} t_{v}=1\right\} \quad \text { and } \\
C H^{0}(A) & =\left\{\sum_{v \in A} t_{v} v: t_{v} \in(0,1) ; \sum_{v \in A} t_{v}=1\right\} .
\end{aligned}
$$

For each $p=1, \ldots, k$, let $e_{p}$ be the $p$ th coordinate vector in $\mathbb{R}^{k}$. Put $e_{0}=0 \in \mathbb{R}^{k}$. Denote by

$$
\Delta^{k}=\mathrm{CH}\left(e_{0}, e_{1}, \ldots, e_{k}\right) \quad \text { and } \quad \operatorname{Int} \Delta^{k}=\mathrm{CH}^{0}\left(e_{0}, e_{1}, \ldots, e_{k}\right)
$$

the standard $k$-simplex and its interior. Let

$$
b_{k}=\frac{1}{k+1}\left(\sum_{q=0}^{q=k} e_{q}\right)=\left(\frac{1}{k+1}, \ldots, \frac{1}{k+1}\right) \in \mathbb{R}^{k}
$$

be the barycenter of $\Delta^{k}$.

For each $p=0,1, \ldots, k$, let

$$
\begin{gathered}
\Delta_{p}^{k}=\mathrm{CH}\left(\left\{e_{q}: q \in\{0,1, \ldots, k\}-p\right\}\right) \\
\text { and } \quad \operatorname{Int} \Delta_{p}^{k}=\mathrm{CH}^{0}\left(\left\{e_{q}: q \in\{0,1, \ldots, k\}-p\right\}\right)
\end{gathered}
$$

denote the $p$ th face of $\Delta^{k}$ and its interior. Define a linear map ${ }^{5}$

$$
\iota_{k, p}: \Delta^{k-1} \longrightarrow \Delta_{p}^{k} \subset \Delta^{k} \quad \text { by } \quad \iota_{k, p}\left(e_{q}\right)= \begin{cases}e_{q}, & \text { if } q<p ; \\ e_{q+1}, & \text { if } q \geq p .\end{cases}
$$

We also define a projection map

$$
\tilde{\pi}_{p}^{k}: \Delta^{k}-\left\{e_{p}\right\} \longrightarrow \Delta_{p}^{k} \quad \text { by } \quad \tilde{\pi}_{p}^{k}\left(\sum_{q=0}^{q=k} t_{q} e_{q}\right)=\frac{1}{1-t_{p}}\left(\sum_{q \neq p} t_{q} e_{q}\right) .
$$

Put

$$
b_{k, p}=\iota_{k, p}\left(b_{k-1}\right), \quad b_{k, p}^{\prime}=\frac{1}{k+1}\left(b_{k}+\sum_{q \neq p} e_{q}\right) .
$$

\footnotetext{
${ }^{5} \mathrm{~A}$ map $f: \Delta^{m} \longrightarrow \Delta^{k}$ is called linear if

$$
f\left(t_{0} e_{0}+\ldots+t_{m} e_{m}\right)=t_{0} f\left(e_{0}\right)+\ldots+t_{m} f\left(e_{m}\right)
$$

for all $\left(t_{0}, \ldots, t_{m}\right) \in[0,1]^{m+1}$ such that $t_{0}+\ldots+t_{m}=1$.
} 

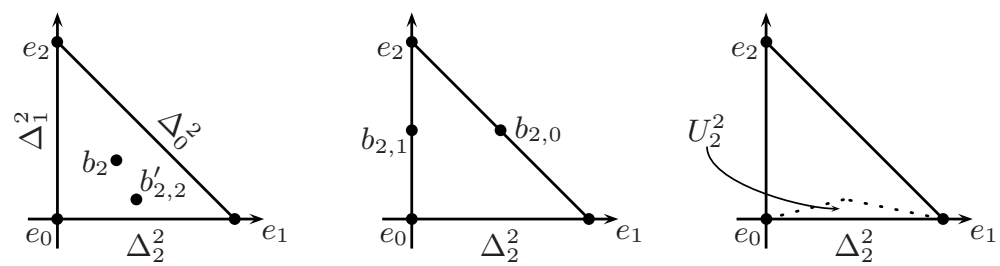

FigurE 1. The standard 2-simplex and some of its distinguished subsets

The points $b_{k, p}$ and $b_{k, p}^{\prime}$ are the barycenters of the $(k-1)$-simplex $\Delta_{p}^{k}$ and of the $k$ simplex spanned by $b_{k}$ and the vertices of $\Delta_{p}^{k}$; see Figure 1 . Define a neighborhood of Int $\Delta_{p}^{k}$ in $\Delta^{k}$ by

$$
U_{p}^{k}=\left\{t_{p} b_{k, p}^{\prime}+\sum_{0 \leq q \leq k ; q \neq p} t_{q} e_{q}: t_{p} \geq 0, t_{q}>0 \forall q \neq p ; \sum_{q=0}^{q=k} t_{q}=1\right\} .
$$

These neighborhoods will be used to construct pseudocycles out of homology cycles.

If $p, q=0,1, \ldots, k$ and $p \neq q$, let

$$
\Delta_{p, q}^{k} \equiv \Delta_{p}^{k} \cap \Delta_{q}^{k}
$$

be the corresponding codimension-two simplex. Define neighborhoods of Int $\Delta_{p, q}^{k}$ in $\Delta^{k}$ by

$$
\begin{gathered}
\tilde{U}_{p, q}^{k}=\left\{t_{p} b_{k, p}+t_{q} b_{k, q}+\sum_{r \neq p, q} t_{r} e_{r}: t_{p}, t_{q} \geq 0, t_{r}>0 \forall r \neq p, q ; \sum_{r=0}^{r=k} t_{r}=1\right\}, \\
U_{p, q}^{k}=\left\{t_{p} \iota_{k, p}\left(b_{\left.k-1, \iota_{k, \iota_{k, p}(q)}^{\prime}\right)}^{\prime}\right)+t_{q} \iota_{k, q}\left(b_{k-1, \iota_{k, \iota}-1, q}^{\prime}\right)+\sum_{r \neq p, q} t_{r} e_{r}:\right. \\
\left.t_{p}, t_{q} \geq 0, t_{r}>0 \forall r \neq p, q ; \sum_{r=0}^{r=k} t_{r}=1\right\} ;
\end{gathered}
$$

see Figure 2. These sets will be used to construct pseudocycle equivalences out of singular chains. Note that

$$
\tilde{U}_{p_{1}, q_{1}}^{k} \cap \tilde{U}_{p_{2}, q_{2}}^{k}=\emptyset \quad \text { if } \quad\left\{p_{1}, q_{1}\right\} \neq\left\{p_{2}, q_{2}\right\} .
$$

Define a projection map

$$
\tilde{\pi}_{p, q}^{k}: \Delta^{k}-\mathrm{CH}\left(e_{p}, e_{q}\right) \longrightarrow \Delta_{p, q}^{k} \quad \text { by } \quad \tilde{\pi}_{p, q}^{k}\left(\sum_{r=0}^{r=k} t_{r} e_{r}\right)=\frac{1}{1-t_{p}-t_{q}}\left(\sum_{r \neq p, q} t_{r} e_{r}\right) \text {. }
$$
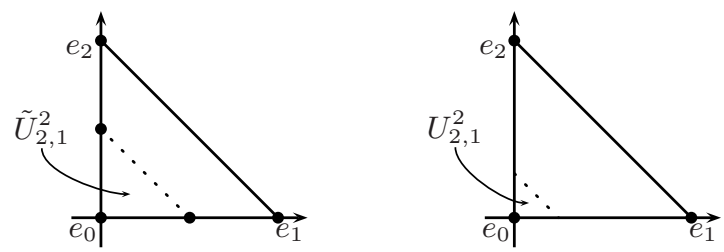

FiguRE 2. The standard 2-simplex and some of its distinguished subsets 
Finally, let $\mathcal{S}_{k}$ denote the group of permutations of the set $\{0, \ldots, k\}$. The set $\mathcal{S}_{k}$ can be viewed as a subset of $\mathcal{S}_{k+1}$; if $\tau \in \mathcal{S}_{k}$, put $\tau(k+1)=k+1$. For any $\tau \in \mathcal{S}_{k}$, let

$$
\tau: \Delta^{k} \longrightarrow \Delta^{k}
$$

be the linear map defined by

$$
\tau\left(e_{q}\right)=e_{\tau(q)} \quad \forall q=0,1, \ldots, k .
$$

Lemma 2.1. If $k \geq 1, Y$ is the $(k-2)$-skeleton of $\Delta^{k}$, and $\tilde{Y}$ is the $(k-2)$-skeleton of $\Delta^{k+1}$, there exist continuous functions

$$
\varphi_{k}: \Delta^{k} \longrightarrow \Delta^{k} \quad \text { and } \quad \tilde{\varphi}_{k+1}: \Delta^{k+1} \longrightarrow \Delta^{k+1}
$$

such that

(i) $\varphi_{k}$ is smooth outside of $Y$ and $\tilde{\varphi}_{k+1}$ is smooth outside of $\tilde{Y}$;

(ii) for all $p=0, \ldots, k$ and $\tau \in \mathcal{S}_{k}$,

$$
\left.\varphi_{k}\right|_{U_{p}^{k}}=\left.\tilde{\pi}_{p}^{k}\right|_{U_{p}^{k}} \quad \text { and } \quad \varphi_{k} \circ \tau=\tau \circ \varphi_{k} ;
$$

(iii) for all $p, q=0, \ldots, k+1$ with $p \neq q$ and $\tilde{\tau} \in \mathcal{S}_{k+1}$,

$$
\begin{gathered}
\left.\tilde{\varphi}_{k+1}\right|_{U_{p, q}^{k+1}}=\left.\tilde{\pi}_{p, q}^{k+1}\right|_{U_{p, q}^{k+1}}, \quad \tilde{\varphi}_{k+1} \circ \tilde{\tau}=\tilde{\tau} \circ \tilde{\varphi}_{k+1}, \\
\text { and } \quad \tilde{\varphi}_{k+1} \circ \iota_{k+1, p}=\iota_{k+1, p} \circ \varphi_{k} .
\end{gathered}
$$

Proof. (1) Choose a smooth function

$$
\tilde{\eta}_{0,1}: \Delta^{k+1}-\Delta_{0,1}^{k+1} \cap \tilde{Y} \longrightarrow[0,1]
$$

such that $\tilde{\eta}_{0,1}=1$ on $U_{0,1}^{k+1}, \tilde{\eta}_{0,1}=0$ outside of $\tilde{U}_{0,1}^{k+1}$, and $\tilde{\eta}_{0,1}$ is invariant under any permutation $\tilde{\tau} \in \mathcal{S}_{k+1}$ that preserves the set $\{0,1\}$. If $\tilde{\tau} \in \mathcal{S}_{k+1}$ is any permutation, let

$$
\tilde{\eta}_{\tilde{\tau}(0), \tilde{\tau}(1)}=\tilde{\eta}_{0,1} \circ \tilde{\tau}^{-1}: \Delta^{k+1}-\Delta_{\tilde{\tau}(0), \tilde{\tau}(1)}^{k+1} \cap \tilde{Y} \longrightarrow[0,1] .
$$

By the assumptions on $\tilde{\eta}_{0,1}, \tilde{\eta}_{p, q}$ is a well-defined smooth function such that $\tilde{\eta}_{p, q}=1$ on $U_{p, q}^{k+1}, \tilde{\eta}_{p, q}=0$ outside of $\tilde{U}_{p, q}^{k+1}$, and

$$
\tilde{\eta}_{\tilde{\tau}(p), \tilde{\tau}(q)}=\tilde{\eta}_{p, q} \circ \tilde{\tau}^{-1}
$$

for all $\tilde{\tau} \in \mathcal{S}_{k+1}$ and distinct $p, q=0, \ldots, k+1$.

(2) Define

$$
\tilde{\varphi}_{k+1}: \Delta^{k+1} \longrightarrow \Delta^{k+1} \quad \text { by } \quad \tilde{\varphi}_{k+1}(x)=x+\sum_{0 \leq p<q \leq k+1} \tilde{\eta}_{p, q}(x) \cdot\left(\tilde{\pi}_{p, q}^{k+1}(x)-x\right) .
$$

Since $\tilde{\eta}_{p, q}$ vanishes on a neighborhood of $\mathrm{CH}\left(e_{p}, e_{q}\right)$ and $\tilde{\pi}_{p, q}^{k+1}$ restricts to the identity on $\Delta_{p, q}^{k+1}$, the function $\tilde{\eta}$ is well-defined, continuous everywhere, and smooth on $\Delta^{k+1}-\tilde{Y}$. By (2.1), $\tilde{\varphi}_{k+1}=\tilde{\pi}_{p, q}^{k+1}$ on $U_{p, q}^{k+1}$. By $(2.4)$, for every $\tilde{\tau} \in \mathcal{S}_{k+1}$

$$
\begin{aligned}
\tilde{\varphi}_{k+1} \circ \tilde{\tau} & =\tilde{\tau}+\sum_{0 \leq p<q \leq k+1} \tilde{\eta}_{p, q} \circ \tilde{\tau} \cdot\left(\tilde{\pi}_{p, q}^{k+1} \circ \tilde{\tau}-\tilde{\tau}\right) \\
& =\tilde{\tau}+\sum_{0 \leq p<q \leq k+1} \tilde{\eta}_{\tilde{\tau}^{-1}(p), \tilde{\tau}^{-1}(q)} \cdot\left(\tilde{\tau} \circ \tilde{\pi}_{\tilde{\tau}^{-1}(p), \tilde{\tau}^{-1}(q)}^{k+1}-\tilde{\tau}\right) \\
& =\tilde{\tau}+\sum_{0 \leq p<q \leq k+1} \tilde{\eta}_{p, q} \cdot\left(\tilde{\tau} \circ \tilde{\pi}_{p, q}^{k+1}-\tilde{\tau}\right)=\tilde{\tau} \circ \tilde{\varphi}_{k+1} .
\end{aligned}
$$

Thus, $\tilde{\varphi}_{k+1}$ satisfies the first two conditions in (2.3), as well as (i) above. 
(3) We define $\varphi_{k}$ by the third condition in (2.3). The function $\varphi_{k}$ is independent of the choice of $p$ and satisfies the second condition in (2.2). To see this, suppose $p, q=0, \ldots, k+1$ and $\tau \in \mathcal{S}_{k}$. Let $\tilde{\tau} \in \mathcal{S}_{k+1}$ be defined by

$$
\tilde{\tau} \circ \iota_{k+1, p}=\iota_{k+1, q} \circ \tau .
$$

If $\varphi_{k, p}$ and $\varphi_{k, q}$ are the functions corresponding to $p$ and $q$ via the third equation in $(2.3)$, then by the second equation in $(2.3)$

$$
\begin{aligned}
\iota_{k+1, q} \circ \tau \circ \varphi_{k, p}=\tilde{\tau} \circ \iota_{k+1, p} \circ \varphi_{k, p} & =\tilde{\tau} \circ \tilde{\varphi}_{k+1} \circ \iota_{k+1, p}=\tilde{\varphi}_{k+1} \circ \tilde{\tau} \circ \iota_{k+1, p} \\
& =\tilde{\varphi}_{k+1} \circ \iota_{k+1, q} \circ \tau=\iota_{k+1, q} \circ \varphi_{k, q} \circ \tau .
\end{aligned}
$$

We conclude that

$$
\tau \circ \varphi_{k, p}=\varphi_{k, q} \circ \tau \quad \forall p, q=0, \ldots, k+1, \tau \in \mathcal{S}_{k} .
$$

The function $\varphi_{k}$ satisfies the first condition in (2.2) because

$$
\begin{gathered}
\iota_{k+1, p}\left(U_{p}^{k}\right)=U_{p, p+1}^{k+1} \cap \Delta_{p, p+1}^{k+1} \quad \text { and } \\
\iota_{k+1, p} \circ \varphi_{k}=\tilde{\varphi}_{k+1} \circ \iota_{k+1, p}=\tilde{\pi}_{p, p+1}^{k+1} \circ \iota_{k+1, p}=\iota_{k+1, p} \circ \tilde{\pi}_{p}^{k} \text { on } U_{p}^{k} .
\end{gathered}
$$

Finally, $\varphi_{k}$ satisfies (i) because $\tilde{\varphi}_{k+1}$ does.

2.2. Homology of neighborhoods of smooth maps. In this subsection, we prove

Proposition 2.2. If $h: Y \longrightarrow X$ is a smooth map and $W$ is an open neighborhood of a subset $A$ of $\operatorname{Im} h$ in $X$, there exists a neighborhood $U$ of $A$ in $W$ such that

$$
H_{l}(U)=0 \quad \text { if } l>\operatorname{dim} Y .
$$

Note that it may not be true that

$$
H_{l}(A)=0 \quad \text { if } l>\operatorname{dim} Y .
$$

For example, let $A$ be the subset of $X=\mathbb{R}^{N}$ consisting of countably many $k$-spheres of radii tending to 0 and having a single point in common. If $k \geq 2$, the set $A$ has infinitely many nonzero homology groups, as shown in [BM].

If $h: Y \longrightarrow X$ is a smooth map and $k$ is a nonnegative integer, put

$$
N_{k}(h)=\left\{y \in Y:\left.\operatorname{rk} d h\right|_{y} \leq k\right\} .
$$

Proposition 2.2 follows from Lemma 2.4 applied with $X$ replaced by $W, Y$ by $h^{-1}(W)$, and $k$ by $\operatorname{dim} Y$.

One of the ingredients in the proof of Lemma 2.4 is Lemma 2.3. For the purposes of this paper, a triangulation of a smooth manifold $X$ is a pair $T=(K, \eta)$ consisting of a simplicial complex and a homeomorphism $\eta:|K| \longrightarrow X$, where $|K|$ is a geometric realization of $K$ in $\mathbb{R}^{N}$ in the sense of Section 3 in [Mu2], such that $\left.\eta\right|_{\text {Int } \sigma}$ is smooth for every simplex $\sigma \in K$.

Lemma 2.3. If $X, Y$ are smooth manifolds and $h: Y \longrightarrow X$ is a smooth map, there exists a triangulation $T=(K, \eta)$ of $X$ such that $h$ is transverse to $\left.\eta\right|_{\text {Int } \sigma}$ for every simplex $\sigma \in K$.

This lemma is clear. In fact, we can start with any triangulation of $X$ and obtain a desired one by an arbitrary small generic perturbation. 
Lemma 2.4. If $h: Y \longrightarrow X$ is a smooth map, for every nonnegative integer $k$ there exists a neighborhood $U$ of $h\left(N_{k}(h)\right)$ in $X$ such that

$$
H_{l}(U)=0 \quad \text { if } l>k \text {. }
$$

Proof. By Lemma 2.3, there exists a triangulation $T=(K, \eta)$ of $X$ such that the smooth map $h$ is transversal to $\left.\eta\right|_{\text {Int } \sigma}$ for all $\sigma \in K$. In particular,

$$
h\left(N_{k}(h)\right) \subset \bigcup_{\sigma \in K, \operatorname{dim} \sigma \geq n-k} \eta(\operatorname{Int} \sigma)=\bigcup_{\sigma \in K, \operatorname{dim} \sigma \geq n-k} \eta\left(\operatorname{St}\left(b_{\sigma}, \operatorname{sd} K\right)\right),
$$

where $n=\operatorname{dim} X$, sd $K$ is the barycentric subdivision of $K$, and $\operatorname{St}\left(b_{\sigma}, \operatorname{sd} K\right)$ is the star of $b_{\sigma}$ in sd $K{ }^{6}$ Note that

$$
\operatorname{St}\left(b_{\sigma}, \operatorname{sd} K\right) \cap \operatorname{St}\left(b_{\sigma^{\prime}}, \operatorname{sd} K\right)=\emptyset
$$

unless $\sigma \subset \sigma^{\prime}$ or $\sigma^{\prime} \subset \sigma$. Furthermore, if $\sigma_{1} \subset \ldots \subset \sigma_{m}$,

$$
\operatorname{St}\left(b_{\sigma_{1}}, \operatorname{sd} K\right) \cap \ldots \cap \operatorname{St}\left(b_{\sigma_{m}}, \operatorname{sd} K\right)=\operatorname{St}\left(b_{\sigma_{1}} \ldots b_{\sigma_{m}}, \operatorname{sd} K\right) ;
$$

the last set is contractable. Put

$$
U_{m}^{\prime}=\bigcup_{\sigma \in K, \operatorname{dim}} \operatorname{St}\left(b_{\sigma}, \operatorname{sd} K\right) .
$$

Then $U_{l_{m}}^{\prime} \cap \ldots \cap U_{m_{j}}^{\prime}$ is a disjoint union of contractable open sets in $|K|$. Let

$$
U_{m}=\eta\left(U_{m}^{\prime}\right), \quad m=n-k, \ldots, n ; \quad U=\bigcup_{m=n-k}^{n} U_{m} .
$$

Since $\eta:|K| \longrightarrow X$ is a homeomorphism, $U_{m_{1}} \cap \ldots \cap U_{m_{j}}$ is a disjoint union of contractable open subsets of $X$. It follows from Lemma 2.5 below that $H_{l}(U)=0$ if $l>k$. Furthermore, by the above $h\left(N_{k}(h)\right) \subset U$.

Lemma 2.5. Let $\left\{U_{m}\right\}_{m=0}^{m=k}$ be a collection of open sets in $X$ and $U=\bigcup_{m=0}^{m=k} U_{m}$. If

$$
H_{l}\left(U_{m_{1}} \cap \ldots \cap U_{m_{j}} ; \mathbb{Z}\right)=0 \quad \forall l>0, m_{1}, \ldots, m_{j}=0, \ldots, k,
$$

then $H_{l}(U)=0$ for all $l>k$.

This lemma follows by induction from Mayer-Vietoris Theorem; see [Mu2, p. 186].

2.3. Oriented homology groups. If $X$ is a simplicial complex, the standard singular chain complex $S_{*}(X)$ most naturally corresponds to the ordered simplicial chain complex of $X$; see Section 13 in [Mu2]. In this subsection, we define a singular chain complex $\bar{S}_{*}(X)$ which corresponds to the standard, or oriented, simplicial chain complex. In particular, its homology is the same as the homology of the ordinary singular chain complex; see Proposition 2.9. On the other hand, it is much easier to construct cycles in $\bar{S}_{*}(X)$ than in $S_{*}(X)$.

If $X$ is a topological space, let $\left(S_{*}(X), \partial_{X}\right)$ denote its singular chain complex, i.e. the free abelian group on the set

$$
\bigcup_{k=0}^{\infty} \operatorname{Hom}\left(\Delta^{k}, X\right)
$$

\footnotetext{
${ }^{6}$ If $K$ is a simplicial complex and $\sigma$ is a simplex in $K$, the star of $\sigma$ in $K$ is the union of the subsets Int $\sigma^{\prime}$ taken over the simplices $\sigma^{\prime} \in K$ such that $\sigma \subset \sigma^{\prime}$; see Section 62 in [Mu2].
} 
of all continuous maps from standard simplices to $X$, along with a map $\partial_{X}$ of degree -1 . Let $S_{k}^{\prime}(X)$ denote the free subgroup of $S_{*}(X)$ spanned by the set

$$
\left\{f-(\operatorname{sign} \tau) f \circ \tau: f \in \operatorname{Hom}\left(\Delta^{k}, X\right) ; \tau \in \mathcal{S}_{k} ; k=0,1, \ldots\right\} .
$$

If $\tau \in \mathcal{S}_{k}$, put

$$
\tilde{\tau}=\operatorname{Id}_{\Delta^{k}}-(\operatorname{sign} \tau) \tau \in S_{k}^{\prime}\left(\Delta^{k}\right) .
$$

Then, $S_{*}^{\prime}(X)$ is the subgroup of $S_{*}(X)$ spanned by

$$
\left\{f_{\#} \tilde{\tau}: f \in \operatorname{Hom}\left(\Delta^{k}, X\right) ; \tau \in \mathcal{S}_{k} ; k=0,1, \ldots\right\} .
$$

Note that if $h: X \longrightarrow Y$ is a continuous map, the linear map

$$
h_{\#}: S_{*}(X) \longrightarrow S_{*}(Y)
$$

maps $S_{*}^{\prime}(X)$ into $S_{*}^{\prime}(Y)$.

Lemma 2.6. The free abelian group $S_{*}^{\prime}(X)$ is a subcomplex of $\left(S_{*}(X), \partial_{X}\right)$, i.e. $\partial_{X} S_{*}^{\prime}(X) \subset S_{*}^{\prime}(X)$.

Proof. (1) Suppose $\tau \in \mathcal{S}_{k}$. For any $p=0, \ldots, k$, let $\tau_{p} \in \mathcal{S}_{k-1}$ be such that

$$
\tau \circ \iota_{k, p}=\iota_{k, \tau(p)} \circ \tau_{p}: \Delta^{k-1} \longrightarrow \Delta_{p}^{k} \subset \Delta^{k} .
$$

Let $\tau_{k, p} \in \mathcal{S}_{k}$ be defined by

$$
\tau_{k, p}(q)= \begin{cases}\iota_{k, p}(q), & \text { if } q<k \\ p, & \text { if } q=k\end{cases}
$$

Then, $\tau \circ \tau_{k, p}=\tau_{k, \tau(p)} \circ \tau_{p} \in \mathcal{S}_{k}$ for all $\tau \in \mathcal{S}_{k}$. Thus,

$$
\operatorname{sign} \tau_{p}=(-1)^{(k-p)+(k-\tau(p))} \operatorname{sign} \quad \tau=(-1)^{p+\tau(p)} \operatorname{sign} \tau .
$$

(2) By the above, we have

$$
\begin{aligned}
\partial_{\Delta^{k}} \tau & =\sum_{p=0}^{k}(-1)^{p} \tau \circ \iota_{k, p}=\sum_{p=0}^{k}(-1)^{p} \iota_{k, \tau(p)} \circ \tau_{p} \\
& =(\operatorname{sign} \tau) \sum_{p=0}^{k}(-1)^{\tau(p)}\left(\operatorname{sign} \tau_{p}\right) \iota_{k, \tau(p)} \circ \tau_{p} \\
& =(\operatorname{sign} \tau) \sum_{p=0}^{k}(-1)^{p}\left(\operatorname{sign} \tau_{\tau^{-1}(p)}\right) \iota_{k, p} \circ \tau_{\tau^{-1}(p)} .
\end{aligned}
$$

Thus,

$$
\partial_{\Delta^{k}} \tilde{\tau}=\sum_{p=0}^{k}(-1)^{p}\left(\iota_{k, p}-\left(\operatorname{sign} \tau_{\tau^{-1}(p)}\right) \iota_{k, p} \circ \tau_{\tau^{-1}(p)}\right) \in S_{k-1}^{\prime}\left(\Delta^{k}\right) .
$$

It follows that for any $f \in S_{k}(X)$,

$$
\partial_{X}\left(f_{\#} \tilde{\tau}\right)=f_{\#}\left(\partial_{\Delta^{k}} \tilde{\tau}\right) \in S_{k-1}^{\prime}(X) .
$$

Lemma 2.7. There exists a natural transformation of functors $D_{X}: S_{*} \longrightarrow S_{*+1}$ such that

(i) if $f: \Delta^{m} \longrightarrow \Delta^{k}$ is a linear map, $D_{X} f$ is a linear combination of linear maps $\Delta^{m+1} \longrightarrow \Delta^{k}$ for all $k, m=0,1, \ldots$;

(ii) $D_{X} S_{*}^{\prime}(X) \subset S_{*}^{\prime}(X)$ for all topological spaces $X$;

(iii) $\partial_{X} D_{X}=(-1)^{k+1} \operatorname{Id}+D_{X} \partial_{X}$ on $S_{k}^{\prime}(X)$. 
Proof. (1) Suppose $k \in \mathbb{Z}^{+}$. If $f: \Delta^{m} \longrightarrow \Delta^{k}$ is a linear map, define a new linear map

$$
P_{k} f: \Delta^{m+1} \longrightarrow \Delta^{k} \quad \text { by } \quad P_{k} f\left(e_{q}\right)= \begin{cases}f\left(e_{q}\right), & \text { if } q=0, \ldots, m \\ b_{k}, & \text { if } q=m+1 .\end{cases}
$$

The transformation $P_{k}$ induces a linear map on the subchain complex of $S_{*}\left(\Delta^{k}\right)$ spanned by the linear maps. If $\tau \in \mathcal{S}_{m} \subset \mathcal{S}_{m+1}$ and $f \in S_{m}\left(\Delta^{k}\right)$, then

$$
P_{k}(f \circ \tau)=P_{k} f \circ \tau \text {. }
$$

Thus, $P_{k}$ maps the subgroup of $S_{*}^{\prime}\left(\Delta^{k}\right)$ spanned by the linear maps into itself. Similarly, if $\tau \in \mathcal{S}_{k}$,

$$
\tau_{\#}\left(P_{k} f\right) \equiv \tau \circ P_{k} f=P_{k}(\tau \circ f) \equiv P_{k}\left(\tau_{\#} f\right) .
$$

Furthermore,

$$
\partial_{\Delta^{k}} P_{k} f=(-1)^{m+1} f+P_{k}\left(\partial_{\Delta^{k}} f\right) .
$$

(2) Let $\left.D_{X}\right|_{S_{k}(X)}=0$ if $k<1$; then $D_{X}$ satisfies (i)-(iii). Suppose $k \geq 1$ and we have defined $\left.D_{X}\right|_{S_{k-1}(X)}$ so that the three requirements are satisfied wherever $D_{X}$ is defined. Put

$$
D_{\Delta^{k}}\left(\operatorname{Id}_{\Delta^{k}}\right)=P_{k}\left(\operatorname{Id}_{\Delta^{k}}+(-1)^{k+1} D_{\Delta^{k}} \partial_{\Delta^{k}} \operatorname{Id}_{\Delta^{k}}\right) \in S_{k+1}\left(\Delta^{k}\right) .
$$

By the inductive assumption (i) and (2.6), $D_{\Delta^{k}}\left(\operatorname{Id}_{\Delta^{k}}\right)$ is a well-defined linear combination of linear maps. For any $f \in \operatorname{Hom}\left(\Delta^{k}, X\right)$, let

$$
D_{X} f=f_{\#} D_{\Delta^{k}} \operatorname{Id}_{\Delta^{k}} .
$$

This construction defines a natural transformation $S_{k} \longrightarrow S_{k+1}$. Since $D_{\Delta^{k}}\left(\operatorname{Id}_{\Delta^{k}}\right)$ is a linear combination of linear maps, it is clear that the requirement (i) above is satisfied; it remains to check (ii) and (iii).

(3) Suppose $f \in \operatorname{Hom}\left(\Delta^{k}, X\right)$ and $\tau \in \mathcal{S}_{k}$; let $s=f_{\#} \tilde{\tau} \in S_{k}^{\prime}(X)$. By (2.11), (2.10), (2.8), and naturality of $\left.D_{X}\right|_{S_{k-1}}$,

$$
\begin{aligned}
D_{X}(f \circ \tau)=f_{\#} \tau_{\#} D_{\Delta^{k}} \operatorname{Id}_{\Delta^{k}} & =f_{\#} \tau_{\#} P_{k}\left(\operatorname{Id}_{\Delta^{k}}+(-1)^{k+1} D_{\Delta^{k}} \partial_{\Delta^{k}} \operatorname{Id}_{\Delta^{k}}\right) \\
& =f_{\#} P_{k}\left(\tau+(-1)^{k+1} \tau_{\#} D_{\Delta^{k}} \partial_{\Delta^{k}} \operatorname{Id}_{\Delta^{k}}\right) \\
& =f_{\#} P_{k}\left(\tau+(-1)^{k+1} D_{\Delta^{k}} \partial_{\Delta^{k}} \tau\right) .
\end{aligned}
$$

Thus,

$$
D_{X} s=f_{\#} P_{k}\left(\tilde{\tau}+(-1)^{k+1} D_{\Delta^{k}} \partial_{\Delta^{k}} \tilde{\tau}\right) .
$$

By Lemma 2.6, the induction assumption (ii), and (2.7), $S_{k}^{\prime}\left(\Delta^{k}\right)$ is mapped into $S_{*}^{\prime}\left(\Delta^{k}\right)$ by $D_{\Delta^{k}} \partial_{\Delta^{k}}$ and by $P_{k}$. Thus, by $(2.13), D_{X}$ maps $S_{k}^{\prime}(X)$ into $S_{k+1}^{\prime}(X)$. Finally, by (2.13), (2.9), and the inductive assumption (iii),

$$
\begin{aligned}
\partial_{X} D_{X} s= & \partial_{X} f_{\#} P_{k}\left(\tilde{\tau}+(-1)^{k+1} D_{\Delta^{k}} \partial_{\Delta^{k}} \tilde{\tau}\right) \\
= & f_{\#} \partial_{\Delta^{k}} P_{k} \tilde{\tau}+(-1)^{k+1} f_{\#} \partial_{\Delta^{k}} P_{k} D_{\Delta^{k}} \partial_{\Delta^{k}} \tilde{\tau} \\
= & f_{\#}\left((-1)^{k+1} \tilde{\tau}+P_{k} \partial_{\Delta^{k}} \tilde{\tau}\right) \\
& \quad+(-1)^{k+1} f_{\#}\left((-1)^{k+1} D_{\Delta^{k}} \partial_{\Delta^{k}} \tilde{\tau}+P_{k} \partial_{\Delta^{k}} D_{\Delta^{k}} \partial_{\Delta^{k}} \tilde{\tau}\right) \\
= & \left((-1)^{k+1} s+D_{X} \partial_{X} s\right)+f_{\#} P_{k} \partial_{\Delta^{k}} \tilde{\tau} \\
& \quad+(-1)^{k+1} f_{\#} P_{k}\left((-1)^{k} \partial_{\Delta^{k}} \tilde{\tau}+D_{\Delta^{k}} \partial_{\Delta^{k}}^{2} \tilde{\tau}\right) \\
= & (-1)^{k+1} s+D_{X} \partial_{X} s .
\end{aligned}
$$


Corollary 2.8. All homology groups of the complex $\left(S_{*}^{\prime}(X),\left.\partial_{X}\right|_{S_{*}^{\prime}(X)}\right)$ are zero.

Let $\bar{S}_{*}(X)=S_{*}(X) / S_{*}^{\prime}(X)$ and denote by

$$
\pi: S_{*}(X) \longrightarrow \bar{S}_{*}(X)
$$

the projection map. Let $\bar{\partial}_{X}$ be boundary map on $\bar{S}_{*}(X)$ induced by $\partial_{X}$. We denote by $\bar{H}_{*}(X ; \mathbb{Z})$ the homology groups of $\left(\bar{S}_{*}(X), \bar{\partial}_{X}\right)$.

Proposition 2.9. If $X$ is a topological space, the projection $\pi: S_{*}(X) \longrightarrow \bar{S}_{*}(X)$ induces a natural isomorphism $H_{*}(X ; \mathbb{Z}) \longrightarrow \bar{H}_{*}(X ; \mathbb{Z})$. This isomorphism can be extended to relative homologies to give an isomorphism of homology theories.

The first statement follows from the long exact sequence in homology for the short exact sequence of chain complexes

$$
0 \longrightarrow S_{*}^{\prime}(X) \longrightarrow S_{*}(X) \stackrel{\pi}{\longrightarrow} \bar{S}_{*}(X) \longrightarrow 0
$$

and Corollary 2.8. The second statement follows from the first and the Five Lemma; see Lemma 24.3 in [Mu2].

For a simplicial complex $K$, there is a natural chain map from the ordered simplicial complex $C_{*}^{\prime}(K)$ to the singular chain complex $S_{*}(|K|)$, which induces isomorphism in homology. If the vertices of $K$ are ordered, there is also a chain map from $C_{*}^{\prime}(K)$ to the oriented chain complex $C_{*}(K)$, which induces a natural isomorphism in homology. However, the chain map itself depends on the ordering of the vertices; see Section 34 in [Mu2]. The advantage of the complex $\bar{S}_{*}(K)$ is that there is a natural chain map from $C_{*}(K)$ to $\bar{S}_{*}(K)$, which induces isomorphism in homology; this chain map is induced by the natural chain map from $C_{*}^{\prime}(K)$ to $S_{*}(|K|)$ described in Section 34 of [Mu2].

If $(X, \mathrm{Bd} X)$ is a compact oriented $n$-manifold, $\left(K, K^{\prime}, \eta\right)$ a triangulation of $(X, \mathrm{Bd} X)$, and for each $n$-dimensional simplex $\sigma \in K$,

$$
l_{\sigma}: \Delta^{n} \longrightarrow \sigma
$$

is a linear map such that $\eta \circ l_{\sigma}$ is orientation-preserving, then the fundamental homology class $[X] \in H_{n}(X, \mathrm{Bd} X)$ is represented in $\bar{S}_{k}(X, \mathrm{Bd} X)$ by

$$
\sum_{\sigma \in K, \operatorname{dim}}\left\{\eta \circ l_{\sigma}\right\} \equiv \sum_{\sigma \in K, \operatorname{dim} \sigma=n} \pi\left(\eta \circ l_{\sigma}\right)
$$

where $\pi$ is as before. Note that

$$
\sum_{\sigma \in K, \operatorname{dim} \sigma=n} \eta \circ l_{\sigma}
$$

may not even be a cycle in $S_{k}(X, \mathrm{Bd} X)$. It is definitely not a cycle if $\mathrm{Bd} X=\emptyset$ and $n$ is an even positive integer, as the boundary of each term $\eta \circ l_{\sigma}$ contains one more term with coefficient +1 than -1 . Similarly, if

$$
h:(X, \mathrm{Bd} X) \longrightarrow(M, U)
$$

is a continuous map, $h_{*}([X]) \in H_{k}(M, U)$ is represented in $\bar{S}_{k}(M, U)$ by

$$
\sum_{\sigma \in K, \operatorname{dim}}\left\{h \circ \eta \circ l_{\sigma=n}\right\} .
$$

Once again, the obvious preimage under $\pi$ of the above chain in $S_{k}(M, U)$ may not even be a cycle. 
2.4. Combinatorics of oriented singular homology. In this subsection we characterize cycles and boundaries in $\bar{S}_{*}(X)$ in a manner suitable for converting them to pseudocycles and pseudocycle equivalences in Subsection 3.1. We will use the two lemmas below to glue maps from standard simplices together to construct smooth maps from smooth manifolds.

The homology groups of a smooth manifold $X$ can be defined with the space $\operatorname{Hom}\left(\Delta^{k}, X\right)$ of continuous maps from $\Delta^{k}$ to $X$ replaced by the space $C^{\infty}\left(\Delta^{k}, X\right)$ of smooth maps; this is a standard fact in differential topology. Note that the operator $D_{X}$ of Lemma 2.7 maps smooth maps into linear combinations of smooth maps. Thus, all of the constructions of Subsection 2.3 go through for the chain complexes based on elements in $C^{\infty}\left(\Delta^{k}, X\right)$ instead of $\operatorname{Hom}\left(\Delta^{k}, X\right)$. Below $\bar{S}_{*}(X)$ will refer to the quotient complex based on such maps.

If $s=\sum_{j=1}^{j=N} f_{j}$, where $f_{j}: \Delta^{k} \longrightarrow X$ is a continuous map for each $j$, let

$$
\mathcal{C}_{s}=\{(j, p): j=1, \ldots, N ; p=0, \ldots, k\}
$$

Lemma 2.10. If $k \geq 1$ and $s \equiv \sum_{j=1}^{j=N} f_{j}$ determines a cycle in $\bar{S}_{k}(X)$, there exist a subset $\mathcal{D}_{s} \subset \mathcal{C}_{s} \times \mathcal{C}_{s}$ disjoint from the diagonal and a map

$$
\tau: \mathcal{D}_{s} \longrightarrow \mathcal{S}_{k-1}, \quad\left(\left(j_{1}, p_{1}\right),\left(j_{2}, p_{2}\right)\right) \longrightarrow \tau_{\left(j_{1}, p_{1}\right),\left(j_{2}, p_{2}\right)},
$$

such that

(i) if $\left(\left(j_{1}, p_{1}\right),\left(j_{2}, p_{2}\right)\right) \in \mathcal{D}_{s}$, then $\left(\left(j_{2}, p_{2}\right),\left(j_{1}, p_{1}\right)\right) \in \mathcal{D}_{s}$;

(ii) the projection $\mathcal{D}_{s} \longrightarrow \mathcal{C}_{s}$ on either coordinate is a bijection;

(iii) for all $\left(\left(j_{1}, p_{1}\right),\left(j_{2}, p_{2}\right)\right) \in \mathcal{D}_{s}$,

$$
\begin{gathered}
\tau_{\left(j_{2}, p_{2}\right),\left(j_{1}, p_{1}\right)}=\tau_{\left(j_{1}, p_{1}\right),\left(j_{2}, p_{2}\right)}^{-1}, \quad f_{j_{2}} \circ \iota_{k, p_{2}}=f_{j_{1}} \circ \iota_{k, p_{1}} \circ \tau_{\left(j_{1}, p_{1}\right),\left(j_{2}, p_{2}\right)}, \\
\text { and } \quad \operatorname{sign} \tau_{\left(j_{1}, p_{1}\right),\left(j_{2}, p_{2}\right)}=-(-1)^{p_{1}+p_{2}} .
\end{gathered}
$$

This lemma follows from the assumption that $\bar{\partial}\{s\}=0$ and from the definition of $\bar{S}_{*}(X)$ in Subsection 2.3. The terms appearing in the boundary of $s$ are indexed by the set $\mathcal{C}_{s}$, and the coefficient of the $(j, p)$ th term is $(-1)^{p}$. Since $s$ determines a cycle in $\bar{S}_{*}(X)$, these terms cancel in pairs, possibly after composition with an element $\tau \in \mathcal{S}_{k-1}$ and multiplying by $\operatorname{sign} \tau$. This operation does not change the equivalence class of a $(k-1)$-simplex in $\bar{S}_{k-1}(X)$.

Lemma 2.11. Suppose $k \geq 1$,

$$
\begin{gathered}
s_{0} \equiv \sum_{j=1}^{j=N_{0}}\left\{f_{0, j}\right\}, \quad s_{1} \equiv \sum_{j=1}^{j=N_{1}}\left\{f_{1, j}\right\}, \quad \tilde{s} \equiv \sum_{j=1}^{j=\tilde{N}} \tilde{f}_{j}, \\
\text { and } \bar{\partial}\{\tilde{s}\}=\left\{s_{1}\right\}-\left\{s_{0}\right\} \in \bar{S}_{k}(X) .
\end{gathered}
$$

Then there exist a subset $\mathcal{D}_{\tilde{s}} \subset \mathcal{C}_{\tilde{s}} \times \mathcal{C}_{\tilde{s}}$ disjoint from the diagonal, subsets $\mathcal{C}_{\tilde{s}}^{(0)}, \mathcal{C}_{\tilde{s}}^{(1)} \subset$ $\mathcal{C}_{\tilde{s}}$, and maps

$$
\begin{gathered}
\tilde{\tau}: \mathcal{D}_{\tilde{s}} \longrightarrow \mathcal{S}_{k}, \quad\left(\left(j_{1}, p_{1}\right),\left(j_{2}, p_{2}\right)\right) \longrightarrow \tilde{\tau}_{\left(j_{1}, p_{1}\right),\left(j_{2}, p_{2}\right)}, \\
\left(\tilde{j}_{i}, \tilde{p}_{i}\right):\left\{1, \ldots, N_{i}\right\} \longrightarrow \mathcal{C}_{\tilde{s}}^{(i)}, \text { and } \tilde{\tau}_{i}:\left\{1, \ldots, N_{i}\right\} \longrightarrow \mathcal{S}_{k}, j \longrightarrow \tilde{\tau}_{(i, j)},
\end{gathered}
$$

where $i=0,1$, such that

(i) if $\left(\left(j_{1}, p_{1}\right),\left(j_{2}, p_{2}\right)\right) \in \mathcal{D}_{\tilde{s}}$, then $\left(\left(j_{2}, p_{2}\right),\left(j_{1}, p_{1}\right)\right) \in \mathcal{D}_{\tilde{s}}$; 
(ii) The projection $\mathcal{D}_{\tilde{s}} \longrightarrow \mathcal{C}_{\tilde{s}}$ on either coordinate is a bijection onto the complement of $\mathcal{C}_{\tilde{s}}^{(0)} \cup \mathcal{C}_{\tilde{s}}^{(1)}$;

(iii) for all $\left(\left(j_{1}, p_{1}\right),\left(j_{2}, p_{2}\right)\right) \in \mathcal{D}_{\tilde{s}}$,

$$
\begin{gathered}
\tilde{\tau}_{\left(j_{2}, p_{2}\right),\left(j_{1}, p_{1}\right)}=\tilde{\tau}_{\left(j_{1}, p_{1}\right),\left(j_{2}, p_{2}\right)}^{-1}, \tilde{f}_{j_{2}} \circ \iota_{k+1, p_{2}}=\tilde{f}_{j_{1}} \circ \iota_{k+1, p_{1}} \circ \tilde{\tau}_{\left(j_{1}, p_{1}\right),\left(j_{2}, p_{2}\right)}, \\
\text { and } \quad \operatorname{sign} \tilde{\tau}_{\left(j_{1}, p_{1}\right),\left(j_{2}, p_{2}\right)}=-(-1)^{p_{1}+p_{2}}
\end{gathered}
$$

(iv) for all $i=0,1$ and $j=1, \ldots, N_{i}$,

$$
\tilde{f}_{\tilde{j}_{i}(j)} \circ \iota_{k+1, \tilde{p}_{i}(j)} \circ \tilde{\tau}_{(i, j)}=f_{i, j} \quad \text { and } \quad \operatorname{sign} \tilde{\tau}_{(i, j)}=-(-1)^{i+\tilde{p}_{i}(j)}
$$

(v) $\left(\tilde{j}_{i}, \tilde{p}_{i}\right)$ is a bijection onto $\mathcal{C}_{\tilde{s}}^{(i)}$ for $i=0,1$.

This lemma follows from the assumption that

$$
\bar{\partial}\{\tilde{s}\}=\left\{s_{1}\right\}-\left\{s_{0}\right\} .
$$

The terms making up $\partial \tilde{s}$ are indexed by the set $\mathcal{C}_{\tilde{s}}$. By definition of $\bar{S}_{*}(X)$, there exist disjoint subsets $\mathcal{C}_{\tilde{s}}^{(0)}$ and $\mathcal{C}_{\tilde{s}}^{(1)}$ of $\mathcal{C}_{\tilde{s}}$ such that for each $(j, p) \in \mathcal{C}_{\tilde{s}}^{(1)}$ the $(j, p)$ th term of $\partial \tilde{s}$ equals one of the terms of $s_{i}$, after a composition with some $\tilde{\tau} \in \mathcal{S}_{k}$ and multiplying by $-(-1)^{i} \operatorname{sign} \tilde{\tau}$. The remaining terms of $\mathcal{C}_{\tilde{s}}$ must cancel in pairs, as in the case of Lemma 2.10 .

\section{INTEGRAL HOMOLOGY AND PSEUDOCYCLES}

3.1. From integral cycles to pseudocycles. In this subsection, we prove

Proposition 3.1. If $X$ is a smooth manifold, there exists a homomorphism

$$
\Psi_{*}: H_{*}(X ; \mathbb{Z}) \longrightarrow \mathcal{H}_{*}(X),
$$

which is natural with respect to smooth maps.

In the proof of Lemma 3.2, we construct a homomorphism from the subgroup of cycles in $\bar{S}_{*}(X)$ to $\mathcal{H}_{*}(X)$. Starting with a cycle $\{s\}$ as in Lemma 2.10, we will glue the functions $f_{j} \circ \varphi_{k}$ together, where $\varphi_{k}$ is the self-map of $\Delta^{k}$ provided by Lemma 2.1. These functions continue to satisfy the second equation in (2.14), i.e.

$$
f_{j_{2}} \circ \varphi_{k} \circ \iota_{k, p_{2}}=f_{j_{1}} \circ \varphi_{k} \circ \iota_{k, p_{1}} \circ \tau_{\left(j_{1}, p_{1}\right),\left(j_{2}, p_{2}\right)} \quad \forall\left(\left(j_{1}, p_{1}\right),\left(j_{2}, p_{2}\right)\right) \in \mathcal{D}_{s},
$$

because $\varphi_{k}=$ id on $\Delta^{k}$-Int $\Delta^{k}$ by the first equation in (2.2). The proof of Lemma 3.2 implements a construction suggested in Section 7.1 of [McSa].

Lemma 3.3 shows that the map of Lemma 3.2 descends to the homology groups. Starting with a chain $\{\tilde{s}\}$ as in Lemma 2.11 , we will glue the functions $\tilde{f}_{j} \circ \tilde{\varphi}_{k+1} \circ \varphi_{k+1}$ together, where $\tilde{\varphi}_{k+1}$ and $\varphi_{k+1}$ are the self-maps of $\Delta^{k+1}$ provided by Lemma 2.1. If $i=0,1$ and $j=1, \ldots, N_{i}$, by the third equation in (2.3), the second equation in (2.2), and the first equation in (2.18)

$$
\begin{aligned}
\tilde{f}_{\tilde{j}_{i}(j)} \circ \tilde{\varphi}_{k+1} \circ \iota_{k+1, \tilde{p}_{i}(j)} \circ \tilde{\tau}_{(i, j)} & =\tilde{f}_{\tilde{j}_{i}(j)} \circ \iota_{k+1, \tilde{p}_{i}(j)} \circ \varphi_{k} \circ \tilde{\tau}_{(i, j)} \\
& =\tilde{f}_{\tilde{j}_{i}(j)} \circ \iota_{k+1, \tilde{p}_{i}(j)} \circ \tilde{\tau}_{(i, j)} \circ \varphi_{k}=f_{i, j} \circ \varphi_{k} .
\end{aligned}
$$

Since $\varphi_{k+1}=$ id on $\Delta^{k+1}-\operatorname{Int} \Delta^{k+1}$, it follows that

$$
\tilde{f}_{\tilde{j}_{i}(j)} \circ \tilde{\varphi}_{k+1} \circ \varphi_{k+1} \circ \iota_{k+1, \tilde{p}_{i}(j)} \circ \tilde{\tau}_{(i, j)}=f_{i, j} \circ \varphi_{k} \quad \forall j=1, \ldots, N_{i}, i=0,1 .
$$


Similarly, if $\left(\left(j_{1}, p_{1}\right),\left(j_{2}, p_{2}\right)\right) \in \mathcal{D}_{\tilde{s}}$, by the third equation in $(2.3)$ used twice, the second equation in (2.16), and the second equation in (2.2),

$$
\begin{aligned}
\tilde{f}_{j_{2}} \circ \tilde{\varphi}_{k+1} \circ \iota_{k+1, p_{2}} & =\tilde{f}_{j_{2}} \circ \iota_{k+1, p_{2}} \circ \varphi_{k}=\tilde{f}_{j_{1}} \circ \iota_{k+1, p_{1}} \circ \tilde{\tau}_{\left(j_{1}, p_{1}\right),\left(j_{2}, p_{2}\right)} \circ \varphi_{k} \\
& =\tilde{f}_{j_{1}} \circ \iota_{k+1, p_{1}} \circ \varphi_{k} \circ \tilde{\tau}_{\left(j_{1}, p_{1}\right),\left(j_{2}, p_{2}\right)} \\
& =\tilde{f}_{j_{1}} \circ \tilde{\varphi}_{k+1} \circ \iota_{k+1, p_{1}} \circ \tilde{\tau}_{\left(j_{1}, p_{1}\right),\left(j_{2}, p_{2}\right)} .
\end{aligned}
$$

Since $\varphi_{k+1}=$ id on $\Delta^{k+1}-$ Int $\Delta^{k+1}$, it follows that

$$
\tilde{f}_{j_{2}} \circ \tilde{\varphi}_{k+1} \circ \varphi_{k+1} \circ \iota_{k+1, p_{2}}=\tilde{f}_{j_{1}} \circ \tilde{\varphi}_{k+1} \circ \varphi_{k+1} \circ \iota_{k+1, p_{1}} \circ \tilde{\tau}_{\left(j_{1}, p_{1}\right),\left(j_{2}, p_{2}\right)}
$$

for all $\left(\left(j_{1}, p_{1}\right),\left(j_{2}, p_{2}\right)\right) \in \mathcal{D}_{\tilde{s}}$. Thus, the functions $\tilde{f}_{j} \circ \tilde{\varphi}_{k+1} \circ \varphi_{k+1}$ are the analogues (in the sense of Lemma 2.11) of the functions $\tilde{f}_{j}$ for the maps $f_{0, j} \circ \varphi_{k}$ and $f_{1, j} \circ \varphi_{k}$.

Lemma 3.2. If $X$ is a smooth manifold, every integral $k$-cycle in $X$, based on $C^{\infty}\left(\Delta^{k} ; X\right)$, determines an element of $\mathcal{H}_{k}(X)$.

Proof. (1) If $k=0$, this is obvious. Suppose $k \geq 1$ and

$$
s \equiv \sum_{j=1}^{j=N} f_{j}
$$

determines a cycle in $\bar{S}_{k}(X)$. Let $\mathcal{D}_{s}$ be the set provided by Lemma 2.10 and let $\tau: \mathcal{D}_{s} \longrightarrow \mathcal{S}_{k-1}$ be the corresponding map. Let

$$
\begin{gathered}
M^{\prime}=\left(\bigsqcup_{j=1}^{j=N}\{j\} \times \Delta^{k}\right) / \sim, \quad \text { where } \\
\left(j_{1}, \iota_{k, p_{1}}\left(\tau_{\left(j_{1}, p_{1}\right),\left(j_{2}, p_{2}\right)}(t)\right)\right) \sim\left(j_{2}, \iota_{k, p_{2}}(t)\right) \quad \forall\left(\left(j_{1}, p_{1}\right),\left(j_{2}, p_{2}\right)\right) \in \mathcal{D}_{s}, t \in \Delta^{k-1} .
\end{gathered}
$$

Let $\pi$ be the quotient map. Define

$$
F: M^{\prime} \longrightarrow X \quad \text { by } \quad F([j, t])=f_{j}\left(\varphi_{k}(t)\right) .
$$

This map is well-defined by (3.1) and continuous by the universal property of the quotient topology; see Theorem 22.2 in [Mu1]. Let $M$ be the complement in $M^{\prime}$ of the set

$$
\pi\left(\bigsqcup_{j=1}^{j=N}\{j\} \times Y\right)
$$

where $Y$ is the $(k-2)$-skeleton of $\Delta^{k}$. By continuity of $F$, compactness of $M^{\prime}$, and the first equation in $(2.2)$,

$$
\left.\mathrm{Bd} F\right|_{M}=F\left(M^{\prime}-M\right)=\bigcup_{j=1}^{j=N} f_{j}\left(\varphi_{k}(Y)\right)=\bigcup_{j=1}^{j=N} f_{j}(Y) .
$$

Since $\left.f_{j}\right|_{\text {Int } \sigma}$ is smooth for all $j=1, \ldots, N$ and all simplices $\sigma \subset \Delta^{k}$, Bd $\left.F\right|_{M}$ has dimension at most $k-2$ by (3.5). Thus, $\left.F\right|_{M}$ is a $k$-pseudocycle, provided $M$ is a smooth oriented manifold and $\left.F\right|_{M}$ is a smooth map. This is shown in (2) below. 
(2) Let $[j, t] \in M$ be any point. If $t \in \operatorname{Int} \Delta^{k}$, then $\pi\left(\{j\} \times \operatorname{Int} \Delta^{k}\right)$ is an open set about $[j, t]$, which is naturally homeomorphic to Int $\Delta^{k}$. If

$$
[j, t]=\left[j_{1}, \iota_{k, p_{1}}\left(t_{1}\right)\right]=\left[j_{2}, \iota_{k, p_{2}}\left(t_{2}\right)\right]
$$

with $\left(j_{1}, p_{1}\right) \neq\left(j_{2}, p_{2}\right)$ and $t_{1} \in \operatorname{Int} \Delta^{k-1}$, let

$$
U=\pi\left(\left\{j_{1}\right\} \times U_{p_{1}}^{k}\right) \cup \pi\left(\left\{j_{2}\right\} \times U_{p_{2}}^{k}\right) .
$$

This is an open neighborhood of $[j, t]$ in $M$. It is homeomorphic in a canonical way to the disjoint union of $U_{p_{1}}^{k}$ and $U_{p_{2}}^{k}$ with Int $\Delta_{p_{1}}^{k} \subset U_{p_{1}}^{k}$ and Int $\Delta_{p_{2}}^{k} \subset U_{p_{2}}^{k}$ identified by the linear map

$$
\iota_{k, p_{1}} \circ \tau_{\left(j_{1}, p_{1}\right),\left(j_{2}, p_{2}\right)} \circ \iota_{k, p_{2}}^{-1}: \operatorname{Int} \Delta_{p_{2}}^{k} \longrightarrow \operatorname{Int} \Delta_{p_{1}}^{k}
$$

and thus to an open subset of $\mathbb{R}^{k}$. By (2.15), the transition map (3.6) is orientationreversing if the open simplices Int $\Delta_{p_{1}}^{k}$ and Int $\Delta_{p_{2}}^{k}$ are oriented as boundaries of the $k$-manifolds $U_{p_{1}}^{k}$ and $U_{p_{2}}^{k}$ with their natural orientations. This means that the induced orientations of $T_{p} U$ coming from the two $k$-manifolds with boundary agree. On any nonempty overlap of this coordinate chart with any other coordinate chart, the transition map is the identity map on an open subset of Int $\Delta^{k}$. Thus, $M$ is a smooth oriented manifold. The map $F$ is smooth on $\{j\} \times \operatorname{Int} \Delta^{k}$ for all $j$ by our assumptions on $F$. If

$$
[j, t]=\left[j_{1}, \iota_{k, p_{1}}\left(t_{1}\right)\right]=\left[j_{2}, \iota_{k, p_{2}}\left(t_{2}\right)\right],
$$

then $F$ is smooth on the open set $U$, defined as above, because it is smooth on

$$
\pi\left(\left\{j_{1}\right\} \times U_{p_{1}}^{k}\right) \text { and } \pi\left(\left\{j_{2}\right\} \times U_{p_{2}}^{k}\right),
$$

and all derivatives in the direction normal to $\pi\left(\left\{j_{1}\right\} \times \operatorname{Int} \Delta_{p_{1}}^{k}\right)$ vanish by the first equation in $(2.2)$.

Remark. The pseudocycle $\left.F\right|_{M}$ constructed above depends on the choice of $\mathcal{D}_{s}$ and $\tau$. However, as the next lemma shows, the image of $\left.F\right|_{M}$ in $\mathcal{H}_{k}(X)$ depends only on $[\{s\}]$.

Lemma 3.3. Under the construction of Lemma 3.2, homologous $k$-cycles determine the same equivalence class of pseudocycles in $\mathcal{H}_{k}(X)$.

Proof. (1) If $k=0$, this is obvious. Suppose $k>0$ and

$$
s_{0} \equiv \sum_{j=1}^{j=N_{0}} f_{0, j} \quad \text { and } \quad s_{1} \equiv \sum_{j=1}^{j=N_{1}} f_{1, j}
$$

determine two homologous $k$-cycles in $\bar{S}_{k}(X)$. Let $\mathcal{D}_{s_{0}}$ and $\mathcal{D}_{s_{1}}$ be the sets provided by Lemma 2.10 and let $\tau_{0}$ and $\tau_{1}$ be the corresponding maps into $\mathcal{S}_{k-1}$. Denote by $\left(M_{0}^{\prime}, M_{0}, F_{0}\right)$ and $\left(M_{1}^{\prime}, M_{1}, F_{1}\right)$ the triples constructed in the proof of Lemma 3.2 corresponding to $s_{0}$ and $s_{1}$. Let

$$
\tilde{s}=\sum_{j=1}^{j=\tilde{N}} \tilde{f}_{j} \in S_{k+1}(X)
$$

be such that

$$
\bar{\partial}\{\tilde{s}\}=\left\{s_{1}\right\}-\left\{s_{0}\right\} \in \bar{S}_{k}(X) .
$$

Denote by $\mathcal{C}_{\tilde{s}}^{(0)}, \mathcal{C}_{\tilde{s}}^{(1)}, \mathcal{D}_{\tilde{s}},\left(\tilde{j}_{i}, \tilde{p}_{i}, \tilde{\tau}_{i}\right)$, and $\tilde{\tau}$ the corresponding objects of Lemma 2.11 . 
Remark. Proceeding as in the proof of Lemma 3.2, we can turn $\tilde{s}$ into a pseudocycle equivalence $\left(\tilde{M}^{*}, \tilde{F}\right)$ between two pseudocycles $\left(M_{0}^{*}, F_{0}\right)$ and $\left(M^{*}, F_{1}\right)$ by gluing across codimension-one faces. Unfortunately, $M_{0}^{*}$ and $M_{1}^{*}$ are not the entire manifolds $M_{0}$ and $M_{1}$; they are missing the $(k-1)$-simplices of $M_{0}$ and $M_{1}$. This issue is resolved in (2) below by adding collars to $\tilde{M}^{*}:(n+1)$-manifolds that begin with $M_{i}^{*}$ and end with $M_{i}^{*}$.

(2) Let $I=[0,1]$. Put

$$
\begin{gathered}
\tilde{M}^{\prime}=\left(\bigsqcup_{j=1}^{j=\tilde{N}}\{j\} \times \Delta^{k+1} \sqcup \bigsqcup_{i=0,1}\{i\} \times I \times M_{i}^{\prime}\right) / \sim, \quad \text { where } \\
\left(j_{1}, \iota_{k+1, p_{1}}\left(\tilde{\tau}_{\left(j_{1}, p_{1}\right),\left(j_{2}, p_{2}\right)}(t)\right)\right) \sim\left(j_{2}, \iota_{k+1, p_{2}}(t)\right) \quad \forall\left(\left(j_{1}, p_{1}\right),\left(j_{2}, p_{2}\right)\right) \in \tilde{\mathcal{D}}_{\tilde{s}}, \\
(i, 1-i, \pi(j, t)) \sim\left(\tilde{j}_{i}(j), \iota_{k+1, \tilde{p}_{i}(j)}\left(\tilde{\tau}_{i, j}(t)\right)\right) \quad \forall j=1, \ldots, N_{i}, i=0,1,
\end{gathered}
$$

for all $t \in \Delta^{k}$. Let

$$
\tilde{\pi}: \bigsqcup_{j=1}^{j=\tilde{N}}\{j\} \times \Delta^{k+1} \sqcup \bigsqcup_{i=0,1}\{i\} \times I \times M_{i}^{\prime} \longrightarrow \tilde{M}^{\prime}
$$

be the quotient map. Define $\tilde{F}: \tilde{M}^{\prime} \longrightarrow X$ by

$$
\begin{array}{ll}
\tilde{F}([j, t])=\tilde{f}_{j}\left(\tilde{\varphi}_{k+1}\left(\varphi_{k+1}(t)\right)\right) & \forall t \in \Delta^{k+1}, j=1, \ldots, \tilde{N} \\
\tilde{F}([i, s, x])=F_{i}(x) & \forall s \in I, x \in M_{i}^{\prime}, i=0,1 .
\end{array}
$$

This map is well-defined by (3.2), (3.3), and (3.4) and is continuous by the universal property of the quotient topology. Let $\tilde{M}$ be the complement in $\tilde{M}^{\prime}$ of the set

$$
\tilde{\pi}\left(\bigsqcup_{j=1}^{j=\tilde{N}}\{j\} \times \tilde{Y} \sqcup \bigsqcup_{i=0,1}\{i\} \times I \times\left(\tilde{M}_{i}^{\prime}-\tilde{M}\right)\right),
$$

where $\tilde{Y}$ is the $(k-1)$-skeleton of $\Delta^{k+1}$. By continuity of $\tilde{F}$, compactness of $\tilde{M}^{\prime}$, and the first equation in (2.3),

$$
\begin{aligned}
\left.\operatorname{Bd} \tilde{F}\right|_{\tilde{M}} & =\tilde{F}\left(\tilde{M}^{\prime}-\tilde{M}\right)=\bigcup_{j=1}^{j=\tilde{N}} \tilde{f}_{j}\left(\tilde{\varphi}_{k+1}\left(\varphi_{k+1}(\tilde{Y})\right)\right) \cup \bigcup_{i=0,1} f_{i, j}\left(\varphi_{k}(Y)\right) \\
& =\bigcup_{j=1}^{j=\tilde{N}} \tilde{f}_{j}(\tilde{Y}) .
\end{aligned}
$$

Since $\left.\tilde{f}_{j}\right|_{\text {Int } \sigma}$ is smooth for all $j=1, \ldots, \tilde{N}$ and all simplices $\sigma \subset \Delta^{k+1},\left.\operatorname{Bd} \tilde{F}\right|_{\tilde{M}}$ has dimension at most $k-1$ by $(3.7)$. Thus, $\left.\tilde{F}\right|_{\tilde{M}}$ is a pseudocycle equivalence between $\left.F_{0}\right|_{M_{0}}$ and $\left.F_{1}\right|_{M_{1}}$, provided $\tilde{M}$ is a smooth oriented manifold, $\left.\tilde{F}\right|_{\tilde{M}}$ is a smooth map, and

$$
\partial\left(\left.\tilde{F}\right|_{\tilde{M}}\right)=\left.F_{1}\right|_{M_{1}}-\left.F_{0}\right|_{M_{0}}
$$

This is shown in (3) below.

(3) By the proof of Lemma 3.2, for each $i=0,1$,

$$
\tilde{M}_{i} \equiv \tilde{M} \cap \tilde{\pi}\left(\{i\} \times I \times M_{i}^{\prime}\right) \approx I \times M_{i}-\{1-i\} \times \bigcup_{j=1}^{j=N_{i}} \pi\left(\{j\} \times\left(\Delta^{k}-\operatorname{Int} \Delta^{k}\right)\right)
$$


is a smooth oriented manifold with boundary,

$$
\partial \tilde{M}_{i} \approx(-1)^{i+1}\left(\{i\} \times M_{i}\right)+(-1)^{i}\left(\{1-i\} \times \bigcup_{j=1}^{j=N_{i}}\{j\} \times \operatorname{Int} \Delta^{k}\right),
$$

and $\tilde{F}$ restricts to a smooth map on $\tilde{M}_{i}$. By the same argument as in (2) of the proof of Lemma 3.2, the space

$$
\tilde{M}^{*} \equiv \tilde{\pi}\left(\bigsqcup_{j=1}^{j=\tilde{N}}\{j\} \times\left(\Delta^{k+1}-\tilde{Y}\right)\right)
$$

is a smooth oriented manifold with boundary,

$$
\partial \tilde{M}^{*} \approx \bigsqcup_{i=0,1} \bigsqcup_{(j, p) \in \mathcal{C}_{\tilde{s}}^{(i)}}\{j\} \times \operatorname{Int} \Delta_{p}^{k+1},
$$

and $\tilde{F}$ restricts to a smooth map on $\tilde{M}^{*}$.

The topological space $\tilde{M}$ is obtained by identifying the $(k+1)$-manifolds $\tilde{M}^{*}$ and $\tilde{M}_{i}$ along components of their boundaries via the map

$$
\begin{gathered}
\{1-i\} \times \bigcup_{j=1}^{j=N_{i}}\{j\} \times \operatorname{Int} \Delta^{k} \longrightarrow \bigsqcup_{(j, p) \in \mathcal{C}_{\tilde{s}}^{(i)}}\{j\} \times \operatorname{Int} \Delta_{p}^{k+1}, \\
(1-i, j, t) \longrightarrow\left(\tilde{j}_{i}(j), \iota_{k+1, \tilde{p}_{i}(j)}(t)\right) .
\end{gathered}
$$

By (2.5) and the second equation in (2.18), this overlap map is orientation-reversing. Therefore, similarly to (2) in the proof of Lemma 3.2 , it follows that $\tilde{M}$ is a smooth oriented manifold with boundary and

$$
\partial \tilde{M}=\tilde{\pi}\left(\{1\} \times\{0\} \times M_{1}\right)-\tilde{\pi}\left(\{0\} \times\{1\} \times M_{0}\right) \approx M_{1}-M_{0} .
$$

It is immediate that under this identification,

$$
\left.\tilde{F}\right|_{M_{i}}=F_{i} \quad \forall i=0,1 .
$$

Finally, as in the proof of Lemma 3.2, all derivatives of the smooth maps $\left.\tilde{F}\right|_{M^{*}}$ and $\left.\tilde{F}\right|_{M_{i}}$ in the normal directions to their boundaries vanish. Therefore, $\left.\tilde{F}\right|_{\tilde{M}}$ is smooth and is a pseudocycle equivalence from $\left.F_{0}\right|_{M_{0}}$ to $\left.F_{1}\right|_{M_{1}}$, as claimed.

3.2. From pseudocycles to integral cycles. In this subsection, we prove

Proposition 3.4. If $X$ is a smooth manifold, there exists a homomorphism

$$
\Phi_{*}: \mathcal{H}_{*}(X) \longrightarrow H_{*}(X ; \mathbb{Z}),
$$

which is natural with respect to smooth maps.

Lemma 3.5. Every $k$-pseudocycle determines a class in $H_{k}(X ; \mathbb{Z})$.

Proof. (1) Suppose $h: M \longrightarrow X$ is a $k$-pseudocycle and $f: N \longrightarrow X$ a smooth map such that

$$
\operatorname{dim} N=k-2 \quad \text { and } \quad \operatorname{Bd} h \subset \operatorname{Im} f .
$$

By Proposition 2.2, there exists an open neighborhood $U$ of $\mathrm{Bd} h$ in $X$ such that

$$
H_{l}(U ; \mathbb{Z})=0 \quad \forall l>k-2 .
$$

Let $K=M-h^{-1}(U)$. Since the closure of $h(M)$ is compact in $X, K$ is a compact subset of $M$ by definition of $\operatorname{Bd} h$. Let $V$ be an open neighborhood of $K$ in $M$ such 
that $\bar{V}$ is a compact manifold with boundary. It inherits an orientation from the orientation of $M$ and thus defines a homology class

$$
[\bar{V}] \in H_{k}(\bar{V}, \operatorname{Bd} \bar{V} ; \mathbb{Z}) \text {. }
$$

Put

$$
[h]=h_{*}([\bar{V}]) \in H_{k}(X, U ; \mathbb{Z}) \approx H_{k}(X ; \mathbb{Z}),
$$

where

$$
h_{*}: H_{k}(\bar{V}, \operatorname{Bd} \bar{V} ; \mathbb{Z}) \longrightarrow H_{k}(X, U ; \mathbb{Z})
$$

is the homology homomorphism induced by $h$. The isomorphism in (3.8) is induced by inclusion. It is an isomorphism by the assumption on the homology of $U$ as follows from the long exact sequence in homology for the pair $(X, U)$.

(2) The homology class $[h]$ is independent of the choice of $V$. Suppose $V^{\prime}$ is another choice such that $\bar{V} \subset V^{\prime}$. Choose a triangulation of $\bar{V}^{\prime}$ extending some triangulation of $(\mathrm{Bd} \bar{V}) \cup\left(\mathrm{Bd} \bar{V}^{\prime}\right)$; such a triangulation exists by Section 16 in [Mu2]. The cycles

$$
h_{*}([\bar{V}]), h_{*}\left(\left[\bar{V}^{\prime}\right]\right) \in H_{k}(X, U ; \mathbb{Z})
$$

then differ by singular simplices lying in $U$; see the discussion at the end of Subsection 2.3. Thus,

$$
h_{*}\left(\left[\bar{V}^{\prime}\right]\right)=h_{*}([\bar{V}]) \in H_{k}(X, U ; \mathbb{Z}) .
$$

(3) The cycle $[h]$ is also independent of the choice of $U$. Suppose $U^{\prime} \subset U$ is another choice. By (2), it can be assumed that $V$ and $V^{\prime}$ chosen as in (1) are the same. Since the isomorphism in (3.8) is the composite of isomorphisms

$$
H_{k}(X ; \mathbb{Z}) \longrightarrow H_{k}\left(X, U^{\prime} ; \mathbb{Z}\right) \longrightarrow H_{k}(X, U ; \mathbb{Z})
$$

induced by inclusions and the homomorphism (3.9) is the composition

$$
H_{k}(\bar{V}, \operatorname{Bd} \bar{V} ; \mathbb{Z}) \longrightarrow H_{k}\left(X, U^{\prime} ; \mathbb{Z}\right) \longrightarrow H_{k}(X, U ; \mathbb{Z}),
$$

the homology classes obtained in $H_{k}(X ; \mathbb{Z})$ from $U$ and $U^{\prime}$ are equal. Finally, if $U$ and $U^{\prime}$ are two arbitrary choices of open sets in (1), by Proposition 2.2 there exists a third choice $U^{\prime \prime} \subset U \cap U^{\prime}$.

Lemma 3.6. Equivalent $k$-pseudocycles determine the same class in $H_{k}(X, \mathbb{Z})$.

Proof. Suppose $h_{i}: M_{i} \longrightarrow X, i=0,1$, are two equivalent $k$-pseudocycles and $\tilde{h}: \tilde{M} \longrightarrow X$ is an equivalence between them. In particular, $\tilde{M}$ is oriented,

$$
\partial \tilde{M}=M_{1}-M_{0}, \quad \text { and }\left.\quad \tilde{h}\right|_{M_{i}}=h_{i} .
$$

Let $\tilde{U}$ be an open neighborhood of $\mathrm{Bd} \tilde{h}$ in $\mathrm{X}$ such that

$$
H_{l}(\tilde{U} ; \mathbb{Z})=0 \quad \forall l>k-1 .
$$

Let $U_{i}$ be an open neighborhood of $\operatorname{Bd} h_{i} \subset \operatorname{Bd} \tilde{h}$ in $\tilde{U}$ such that

$$
H_{l}\left(U_{i} ; \mathbb{Z}\right)=0 \quad \forall l>k-2,
$$

as provided by Proposition 2.2. Let $V_{i} \subset M_{i}$ be a choice of an open set as in (1) of the proof of Lemma 3.5. For $i=0,1$, choose a triangulation of $M_{i}$ that extends a triangulation of $\mathrm{Bd} \bar{V}_{i}$. Extend these two triangulations to a triangulation $\tilde{T}=(\tilde{K}, \tilde{\eta})$ of $\tilde{M}$. Let $K$ be a finite sub-complex of $\tilde{K}$ such that

$$
V_{0}, V_{1} \subset \tilde{\eta}(|K|) \quad \text { and } \quad \tilde{M}-\tilde{h}^{-1}(\tilde{U}) \subset \tilde{\eta}(\operatorname{Int}|K|) .
$$


Such a subcomplex exists because $\tilde{h}(\tilde{M})$ is a pre-compact subset of $X$ and thus $\tilde{M}-\tilde{h}^{-1}(\tilde{U})$ is a compact subset of $\tilde{M}$. Put

$$
K_{i}=\left\{\sigma \in K: \eta(\sigma) \subset \bar{V}_{i}\right\} \quad \text { for } i=0,1 .
$$

By the proof of Lemma 3.5, $\left(K_{i},\left.\tilde{h} \circ \tilde{\eta}\right|_{\left|K_{i}\right|}\right)$ determines the homology class $\left[h_{i}\right] \in$ $H_{k}\left(X, U_{i} ; \mathbb{Z}\right)$. Let $\left[h_{i}^{\prime}\right]$ denote its image in $H_{k}(X, \tilde{U} ; \mathbb{Z})$ under the homomorphism induced by inclusion. The above assumptions on $K$ imply that

$$
\partial\left(K,\left.\tilde{h} \circ \tilde{\eta}\right|_{K}\right)=\left(K_{1},\left.\tilde{h} \circ \tilde{\eta}\right|_{K_{1}}\right)-\left(K_{0},\left.\tilde{h} \circ \tilde{\eta}\right|_{K_{0}}\right)
$$

in $\bar{S}(M, \tilde{U})$. Thus,

$$
\left[h_{0}^{\prime}\right]=\left[h_{1}^{\prime}\right] \in H_{k}(X, \tilde{U} ; \mathbb{Z}),
$$

and this class lies in the image of the homomorphism

$$
H_{k}(X ; \mathbb{Z}) \longrightarrow H_{k}(X, \tilde{U} ; \mathbb{Z})
$$

induced by inclusion. This map is equal to the composites

$$
\begin{aligned}
& H_{k}(X ; \mathbb{Z}) \longrightarrow H_{k}\left(X, U_{0} ; \mathbb{Z}\right) \longrightarrow H_{k}(X, \tilde{U} ; \mathbb{Z}), \\
& H_{k}(X ; \mathbb{Z}) \longrightarrow H_{k}\left(X, U_{1} ; \mathbb{Z}\right) \longrightarrow H_{k}(X, \tilde{U} ; \mathbb{Z}) .
\end{aligned}
$$

Since $H_{k}(\tilde{U} ; \mathbb{Z})=0$, the homomorphism $(3.10)$ is injective. Thus, $\left[h_{0}\right]$ and $\left[h_{1}\right]$ come from the same element of $H_{k}(X ; \mathbb{Z})$.

3.3. Isomorphism of homology theories. In this subsection we conclude the proof of Theorem 1.1.

Lemma 3.7. If $X$ is a smooth manifold, the composition

$$
\Phi_{*} \circ \Psi_{*}: H_{*}(X ; \mathbb{Z}) \longrightarrow \mathcal{H}_{*}(X) \longrightarrow H_{*}(X ; \mathbb{Z})
$$

is the identity map on $H_{*}(X ; \mathbb{Z})$.

Proof. Suppose

$$
\{s\}=\sum_{j=1}^{N}\left\{f_{j}\right\} \in \bar{S}_{k}(X)
$$

is a cycle and $F: M \longrightarrow X$ is a pseudocycle corresponding to $s$ via the construction of Lemma 3.2. Recall that $M$ is the complement of the $(k-2)$-simplices in a compact space $M^{\prime}$ and $F$ is the restriction of a continuous map $F^{\prime}: M^{\prime} \longrightarrow X$ induced by the maps

$$
f_{j} \circ \varphi_{k}: \Delta^{k} \longrightarrow X, \quad j=1, \ldots, N
$$

Since $\varphi_{k}$ is homotopic to the identity on $\Delta^{k}$, with boundary fixed,

$$
f_{j} \circ \varphi_{k}-f_{j} \in \partial S_{k+1}(X) \quad \forall j=1, \ldots, N .
$$

Let $U$ be a neighborhood of $\mathrm{Bd} F$ such that

$$
H_{l}(U ; \mathbb{Z})=0 \quad \forall l>k-2 .
$$

Put $K=M-f^{-1}\left(\varphi_{k}^{-1}(U)\right)$. Let $V$ be a pre-compact neighborhood of $K$ such that $(\bar{V}, \partial \bar{V})$ is a smooth manifold with boundary. Choose a triangulation $T=(K, \eta)$ of $(\bar{V}, \partial \bar{V})$ such that every $k$-simplex of $T$ is contained in a set of the form $\pi\left(\{j\} \times \Delta^{k}\right)$, where $\pi$ is as in the proof of Lemma 3.2. Put

$$
K_{j}=\left\{\sigma \in K: \eta(\sigma) \subset \pi\left(\{j\} \times \Delta^{k}\right)\right\}, \quad K_{j}^{\mathrm{top}}=\left\{\sigma \in K_{j}: \operatorname{dim} \sigma=k\right\} .
$$


Let $\tilde{T}_{j}=\left(\tilde{K}_{j}, \eta_{j}\right)$ be a triangulation of a subset of $\Delta^{k}$ that along with $K_{j}$ gives a triangulation of $\Delta^{k}$. Put

$$
\tilde{K}_{j}^{\text {top }}=\left\{\sigma \in \tilde{K}_{j}: \operatorname{dim} \sigma=k\right\} .
$$

By definition of $T$,

$$
f_{j} \circ \varphi_{k}\left(\eta_{j}(\sigma)\right) \subset U \quad \forall \sigma \in \tilde{K}_{j}^{\text {top }} .
$$

By (3.11), modulo $\bar{\partial} \bar{S}_{k+1}(X)$ we have

$$
\begin{aligned}
\{s\} & =\sum_{\sigma \in K^{\mathrm{top}}}\left\{f_{j} \circ \varphi_{k} \circ \eta \circ l_{\sigma}\right\} \\
& =\sum_{j=1}^{N} \sum_{\sigma \in K_{j}^{\mathrm{top}}}\left\{f_{j} \circ \varphi_{k} \circ \eta \circ l_{\sigma}\right\}+\sum_{j=1}^{N} \sum_{\sigma \in \tilde{K}_{j}^{\mathrm{top}}}\left\{f_{j} \circ \varphi_{k} \circ \tilde{\eta}_{j} \circ l_{\sigma}\right\}
\end{aligned}
$$

since subdivisions of cycles do not change the homology class. By the proof of Lemma 3.5, the first sum on the right-hand side of (3.13) represents $[F]$ in $\bar{S}_{k}(X, U)$. By (3.12), the second sum lies in $\bar{S}_{k}(U)$. Since the sum of the two terms is a cycle in $\bar{S}_{k}(X)$, it must represent $[F]$ in $\bar{S}_{k}(X)$. Thus,

$$
\{F\}=\{s\} \in H_{k}(X ; \mathbb{Z}),
$$

and the claim follows.

Lemma 3.8. If $X$ is a smooth manifold, the homomorphism $\Phi_{*}: \mathcal{H}_{*}(X) \longrightarrow$ $H_{*}(X ; \mathbb{Z})$ is injective.

We will assume that a $k$-pseudocycle $h: M^{\prime} \longrightarrow X$ determines the zero homology class via the construction of Lemma 3.5 and show that it must be the boundary of a smooth map $\tilde{F}: \tilde{M} \longrightarrow X$ in the sense of pseudocycles. A new difficulty here is that $M^{\prime}$ need not be compact, and therefore $\tilde{M}$ may need to be constructed from infinitely many $(k+1)$-simplices. This will be achieved as the limit of finite stages $\tilde{M}_{i}$, so that as $i \in \mathbb{Z}^{+}$increases $M^{\prime}$ will be the pseudocycle boundary of $\tilde{M}_{i}$ "modulo" smaller and smaller neighborhoods $U_{i}$ of $\mathrm{Bd} h$. As in the proof of Lemma 3.3 , we will also need to attach a collar to the $(k+1)$-manifold $\tilde{M}^{*}$ obtained directly from a bounding chain.

Proof. (1) Suppose a $k$-pseudocycle $h: M^{\prime} \longrightarrow X$ determines the zero homology class. It can be assumed that $k \geq 1$; otherwise, there is nothing to prove. Let $\left\{U_{i}\right\}_{i=1}^{\infty}$ be a sequence of open pre-compact neighborhoods of $\operatorname{Bd} h$ in $X$ such that

$$
U_{i+1} \subset U_{i}, \quad \bigcap_{i=1}^{\infty} U_{i}=\operatorname{Bd} h, \quad \text { and } \quad H_{l}\left(U_{i} ; \mathbb{Z}\right)=0 \quad \forall l>k-2 .
$$

Existence of such a collection follows from Proposition 2.2 and metrizability of any manifold. Let $\left\{V_{i}\right\}_{i=1}^{\infty}$ be a corresponding collection of open sets in $M^{\prime}$ as in (1) of the proof of Lemma 3.5. It can be assumed that $\bar{V}_{i} \subset V_{i+1}$. Choose a triangulation $T=(K, \eta)$ of $M^{\prime}$ that extends a triangulation of $\bigcup_{i=1}^{\infty} \operatorname{Bd} \bar{V}_{i}$. Let

$$
K^{\text {top }}=\{\sigma \in K: \operatorname{dim} \sigma=k\}, \quad \mathcal{C}_{\eta}=\left\{(\sigma, p): \sigma \in K^{\text {top }}, p=0,1, \ldots, k\right\} .
$$

For each $\sigma \in K^{\text {top }}$, let

$$
l_{\sigma}: \Delta^{k} \longrightarrow \sigma \subset|K| \subset \mathbb{R}^{\infty}
$$


be a linear map such that $\eta \circ l_{\sigma}$ is orientation-preserving. Put

$$
\begin{gathered}
f_{\sigma}=h \circ \eta \circ l_{\sigma} \quad \forall \sigma \in K^{\text {top }} \quad \text { and } \\
\mathcal{D}_{\eta}=\left\{\left(\left(\sigma_{1}, p_{1}\right),\left(\sigma_{2}, p_{2}\right)\right) \in \mathcal{C}_{\eta} \times \mathcal{C}_{\eta}:\left(\sigma_{1}, p_{1}\right) \neq\left(\sigma_{2}, p_{2}\right), l_{\sigma_{1}}\left(\Delta_{p_{1}}^{k}\right)=l_{\sigma_{2}}\left(\Delta_{p_{2}}^{k}\right)\right\} .
\end{gathered}
$$

For each $\left(\left(\sigma_{1}, p_{1}\right),\left(\sigma_{2}, p_{2}\right)\right) \in \mathcal{D}_{\eta}$, define

$$
\tau_{\left(\sigma_{1}, p_{1}\right),\left(\sigma_{2}, p_{2}\right)} \in \mathcal{S}_{k-1} \quad \text { by } \quad l_{\sigma_{2}} \circ \iota_{k, p_{2}}=l_{\sigma_{1}} \circ \iota_{k, p_{1}} \circ \tau_{\left(\sigma_{1}, p_{1}\right),\left(\sigma_{2}, p_{2}\right)} .
$$

Since $K$ is an oriented simplicial complex,

$$
\mathcal{D}_{\eta} \subset \mathcal{C}_{\eta} \times \mathcal{C}_{\eta} \quad \text { and } \quad \tau: \mathcal{D}_{\eta} \longrightarrow \mathcal{S}_{k-1}
$$

satisfy (i)-(iii) of Lemma 2.10. Furthermore, $M^{\prime}$ is the topological space corresponding to $\left(\mathcal{C}_{\eta}, \mathcal{D}_{\eta}, \tau\right)$ via the construction of Lemma 3.2 and $h$ is the continuous map described by

$$
\left.h\right|_{\pi\left(\sigma \times \Delta^{k}\right)}=f_{\sigma} .
$$

As in the proof of Lemma 3.2, let $M$ be the complement of the $(k-2)$-simplices in $M^{\prime}$; the pseudocycles $h$ and $\left.h\right|_{M}$ are equivalent. Since $\varphi_{k}$ is homotopic to the identity on $\Delta^{k}$ with boundary fixed, the pseudocycle $\left.h\right|_{M}$ is in turn equivalent to the pseudocycle $\left.F\right|_{M}$, where as in the proof of Lemma 3.2

$$
F: M^{\prime} \longrightarrow X, \quad F \circ \eta \circ l_{\sigma}=f_{\sigma} \circ \varphi_{k} .
$$

(2) For each $i \geq 1$, let

$$
\begin{gathered}
K_{i}^{\text {top }}=\left\{\sigma \in K^{\text {top }}: \eta(\sigma) \subset \bar{V}_{i}\right\}, \quad \mathcal{C}_{\eta ; i}=\left\{(\sigma, p) \in \mathcal{C}_{\eta}: \sigma \in K_{i}^{\text {top }}\right\}, \\
\text { and } \quad \mathcal{D}_{\eta ; i}=\mathcal{D}_{\eta} \cap\left(\mathcal{C}_{\eta ; i} \times \mathcal{C}_{\eta ; i}\right) .
\end{gathered}
$$

By construction of $[h]$, for every $i \geq 1$, there exists a singular chain

$$
s_{i} \equiv \sum_{j=1}^{N_{i}} f_{i, j} \in S_{k}\left(U_{i}\right)
$$

such that

$$
\sum_{\sigma \in K_{i}^{\mathrm{top}}}\left\{h \circ \eta \circ l_{\sigma}\right\}+\left\{s_{i}\right\}
$$

is a cycle in $\bar{S}_{k}(X)$ representing $[h]$. Similarly to Lemma 2.10 , there exist a symmetric subset

$$
\mathcal{D}_{i} \subset\left(\mathcal{C}_{\eta ; i} \sqcup \mathcal{C}_{s_{i}}\right) \times\left(\mathcal{C}_{\eta ; i} \sqcup \mathcal{C}_{s_{i}}\right)
$$

disjoint from the diagonal and a map

$$
\tau_{i}: \mathcal{D}_{i} \longrightarrow \mathcal{S}_{k-1}
$$

such that

(i) $\mathcal{D}_{\eta ; i} \subset \mathcal{D}_{i}$ and $\left.\tau_{i}\right|_{\mathcal{D}_{\eta ; i}}=\left.\tau\right|_{\mathcal{D}_{\eta ; i}}$;

(ii) the projection map $\mathcal{D}_{i} \longrightarrow \mathcal{C}_{\eta ; i} \sqcup \mathcal{C}_{s_{i}}$ on either coordinate is a bijection;

(iii) for all $\left(\left(j_{1}, p_{1}\right),\left(j_{2}, p_{2}\right)\right) \in \mathcal{D}_{i}$,

$$
\begin{aligned}
\tau_{\left(j_{2}, p_{2}\right),\left(j_{1}, p_{1}\right)}= & \tau_{\left(j_{1}, p_{1}\right),\left(j_{2}, p_{2}\right)}^{-1}, \quad f_{i, j_{2}} \circ \iota_{k, p_{2}}=f_{i, j_{1}} \circ \iota_{k, p_{1}} \circ \tau_{\left(j_{1}, p_{1}\right),\left(j_{2}, p_{2}\right)}, \\
& \text { and } \operatorname{sign} \tau_{\left(j_{1}, p_{1}\right),\left(j_{2}, p_{2}\right)}=-(-1)^{p_{1}+p_{2}},
\end{aligned}
$$

where $f_{i, \sigma} \equiv f_{\sigma}$ for all $\sigma \in K_{i}^{\text {top }}$. 
(3) By (2), for each $i \geq 2$

$$
\sum_{\sigma \in K_{i}^{\text {top }}-K_{i-1}^{\text {top }}}\left\{h \circ \eta \circ l_{\sigma}\right\}+\left\{s_{i}\right\}-\left\{s_{i-1}\right\} \in \bar{S}_{k}\left(U_{i-1}\right)
$$

is a cycle. Since $H_{k}\left(U_{i-1} ; \mathbb{Z}\right)=0$, it must be a boundary. If $i=1$, this conclusion is still true with $U_{0}=X, K_{0}^{\text {top }}=\emptyset$, and $s_{0}=0$, since $[h]=0$ by assumption. Let

$$
\tilde{s}_{i} \equiv \sum_{j=1}^{\tilde{N}_{i}} \tilde{f}_{i, j} \in S_{k+1}\left(U_{i-1}\right)
$$

be such that

$$
\sum_{\sigma \in K_{i}^{\text {top }}-K_{i-1}^{\text {top }}}\left\{h \circ \eta \circ l_{\sigma}\right\}+\left\{s_{i}\right\}-\left\{s_{i-1}\right\}=\bar{\partial}\left\{\tilde{s}_{i}\right\} \in \bar{S}_{k}\left(U_{i-1}\right) .
$$

Similarly to Lemma 2.11, there exist

$$
\tilde{\mathcal{C}}_{i}^{(0)} \subset \tilde{\mathcal{C}}_{i} \equiv \bigsqcup_{i^{\prime}=1}^{i^{\prime}=i} \mathcal{C}_{\tilde{s}_{i^{\prime}}}
$$

a symmetric subset $\tilde{\mathcal{D}}_{i} \subset \tilde{\mathcal{C}}_{i} \times \tilde{\mathcal{C}}_{i}$ disjoint from the diagonal, and maps

$$
\begin{aligned}
\tilde{\tau}_{i}: \tilde{\mathcal{D}}_{i} \longrightarrow & \mathcal{S}_{k},\left(\left(j_{1}, p_{1}\right),\left(j_{2}, p_{2}\right)\right) \longrightarrow \tilde{\tau}_{i,\left(\left(j_{1}, p_{1}\right),\left(j_{2}, p_{2}\right)\right)}, \\
& \left(\tilde{j}_{i}, \tilde{p}_{i}\right): K_{i}^{\mathrm{top}} \sqcup\left\{1, \ldots, N_{i}\right\} \longrightarrow \tilde{\mathcal{C}}_{i}^{(0)}, \\
\text { and } \quad & \tilde{\tau}_{i}: K_{i}^{\mathrm{top}} \sqcup\left\{1, \ldots, N_{i}\right\} \longrightarrow \mathcal{S}_{k}, j \longrightarrow \tilde{\tau}_{(i, j)},
\end{aligned}
$$

such that

(i) $\tilde{\mathcal{D}}_{i} \subset \tilde{\mathcal{D}}_{i+1},\left.\tilde{\tau}_{i+1}\right|_{\tilde{\mathcal{D}}_{i}}=\tilde{\tau}_{i}$, and $\left.\left(\tilde{j}_{i+1}, \tilde{p}_{i+1}, \tilde{\tau}_{i+1}\right)\right|_{K_{i}^{\text {top }}}=\left.\left(\tilde{j}_{i}, \tilde{p}_{i}, \tilde{\tau}_{i}\right)\right|_{K_{i}^{\text {top }}}$;

(ii) the projection $\tilde{\mathcal{D}}_{i} \longrightarrow \tilde{\mathcal{C}}_{i}$ on either coordinate is a bijection onto the complement of $\tilde{\mathcal{C}}_{i}^{(0)}$;

(iii) for all $\left(\left(j_{1}, p_{1}\right),\left(j_{2}, p_{2}\right)\right) \in \tilde{\mathcal{D}}_{i} \cap\left(\mathcal{C}_{\tilde{s}_{i_{1}}} \times \mathcal{C}_{\tilde{s}_{i_{2}}}\right)$,

$$
\begin{array}{cl}
\tilde{\tau}_{i,\left(\left(j_{2}, p_{2}\right),\left(j_{1}, p_{1}\right)\right)}=\tilde{\tau}_{i,\left(\left(j_{1}, p_{1}\right),\left(j_{2}, p_{2}\right)\right)}^{-1}, \quad \operatorname{sign} \tilde{\tau}_{i,\left(\left(j_{1}, p_{1}\right),\left(j_{2}, p_{2}\right)\right)}=-(-1)^{p_{1}+p_{2}}, \\
\text { and } \tilde{f}_{i_{2}, j_{2}} \circ \iota_{k+1, p_{2}}=\tilde{f}_{i_{1}, j_{1}} \circ \iota_{k+1, p_{1}} \circ \tilde{\tau}_{i,\left(\left(j_{1}, p_{1}\right),\left(j_{2}, p_{2}\right)\right)} ;
\end{array}
$$

(iv) for all $\sigma \in K_{i}^{\mathrm{top}}-K_{i-1}^{\mathrm{top}}$,

$$
\tilde{f}_{i, \tilde{j}_{i}(j)} \circ \iota_{k+1, \tilde{p}_{i}(j)} \circ \tilde{\tau}_{(i, j)}=f_{\sigma} \quad \text { and } \quad \operatorname{sign} \tilde{\tau}_{(i, j)}=-(-1)^{\tilde{p}_{i}(j)} ;
$$

(v) $\left(\tilde{j}_{i}, \tilde{p}_{i}\right)$ is a bijection onto $\tilde{\mathcal{C}}_{i}^{(0)}$.

(4) Put

$$
\tilde{M}^{\prime}=\left(\bigsqcup_{i=1}^{\infty} \bigsqcup_{j=1}^{\tilde{N}_{i}}\{i\} \times\{j\} \times \Delta^{k+1} \sqcup I \times M^{\prime}\right) / \sim,
$$

where for all $t \in \Delta^{k}$

$$
\begin{gathered}
\left(i_{1}, j_{1}, \iota_{k, p_{1}}\left(\tilde{\tau}_{i,\left(\left(j_{1}, p_{1}\right),\left(j_{2}, p_{2}\right)\right)}(t)\right)\right) \sim\left(i_{2}, j_{2}, \iota_{k, p_{2}}(t)\right) \\
\forall\left(\left(j_{1}, p_{1}\right),\left(j_{2}, p_{2}\right)\right) \in \tilde{\mathcal{D}}_{i} \cap\left(\mathcal{C}_{\tilde{s}_{i_{1}}} \times \mathcal{C}_{\tilde{s}_{i_{2}}}\right), \\
(1, \pi(\sigma, t)) \sim\left(i, \tilde{j}_{i}(\sigma), \iota_{k+1, \tilde{p}_{i}(\sigma)}\left(\tilde{\tau}_{i, \sigma}(t)\right)\right) \quad \forall \sigma \in K_{i}^{\mathrm{top}}-K_{i-1}^{\mathrm{top}}, i \in \mathbb{Z}^{+} .
\end{gathered}
$$


Let

$$
\tilde{\pi}: \bigsqcup_{i=1}^{\infty} \bigsqcup_{j=1}^{\tilde{N}_{i}}\{i\} \times\{j\} \times \Delta^{k+1} \sqcup I \times M^{\prime} \longrightarrow \tilde{M}^{\prime}
$$

be the quotient map. Define $\tilde{F}: \tilde{M}^{\prime} \longrightarrow X$ by

$$
\begin{array}{ll}
\tilde{F}([i, j, t])=\tilde{f}_{i, j}\left(\tilde{\varphi}_{k+1}\left(\varphi_{k+1}(t)\right)\right) & \forall t \in \Delta^{k+1}, j=1, \ldots, \tilde{N}_{i}, i \in \mathbb{Z}^{+} ; \\
\tilde{F}[s, x]=F(x) & \forall s \in I, x \in M^{\prime},
\end{array}
$$

where $\tilde{\varphi}_{k+1}$ and $\varphi_{k+1}$ are the self-maps of $\Delta^{k+1}$ provided by Lemma 2.1. Similarly to the proof of Lemma 3.3, this map is well-defined and continuous. Since the image of

$$
\bigsqcup_{i=2}^{\infty} \bigsqcup_{j=1}^{\tilde{N}_{i}}\{i\} \times\{j\} \times \Delta^{k+1} \sqcup I \times \pi\left(\bigsqcup_{i=2}^{\infty} \bigsqcup_{\sigma \in K_{2}^{\text {top }}}\{\sigma\} \times \Delta^{k-1}\right)
$$

under $\tilde{F} \circ \tilde{\pi}$ is contained in the pre-compact subset $U_{1}$ of $X, \tilde{F}\left(\tilde{M}^{\prime}\right)$ is a pre-compact subset of $X$ as well.

Let $\tilde{M}$ be the complement in $\tilde{M}^{\prime}$ of the set

$$
\tilde{\pi}\left(\bigsqcup_{i=1}^{\infty} \bigsqcup_{j=1}^{j=\tilde{N}_{i}}\{i\} \times\{j\} \times \tilde{Y} \sqcup I \times\left(M^{\prime}-M\right)\right),
$$

where $\tilde{Y} \subset \Delta^{k+1}$ is the $(k-1)$-skeleton. Similarly to the proof of Lemma $3.3,\left.\operatorname{Bd} \tilde{F}\right|_{\tilde{M}}$ is of dimension at most $k-1, \tilde{M}$ is a smooth oriented manifold boundary,

$$
\partial \tilde{M}=-M,
$$

$\left.\tilde{F}\right|_{\tilde{M}}$ is a smooth map, and $\left.\tilde{F}\right|_{M}=\left.F\right|_{M}$. We note that in this case,

$$
\begin{aligned}
\tilde{M}_{0} & =I \times M-\{1\} \times \bigcup_{\sigma \in K_{i}^{\text {top }}} \pi\left(\{\sigma\} \times\left(\Delta^{k}-\operatorname{Int} \Delta^{k}\right)\right), \\
\partial \tilde{M}_{0} & \approx-\{0\} \times M+\{1\} \times \bigsqcup_{\sigma \in K^{\text {top }}}\{\sigma\} \times \operatorname{Int} \Delta^{k}, \\
\tilde{M}^{*} & =\tilde{\pi}\left(\bigsqcup_{i=1}^{\infty} \bigsqcup_{j=1}^{j=\tilde{N}_{i}}\{i\} \times\{j\} \times\left(\Delta^{k+1}-\tilde{Y}\right)\right) \\
\partial \tilde{M}^{*} & =\tilde{\pi}\left(\bigsqcup_{i=1}^{\infty} \bigsqcup_{\sigma \in K_{i}^{\text {top }}}\{i\} \times\left\{\tilde{j}_{i}(\sigma)\right\} \times \operatorname{Int} \Delta_{\tilde{p}_{i}(\sigma)}^{k+1}\right) .
\end{aligned}
$$

The topological space $\tilde{M}$ is obtained from $\tilde{M}^{*}$ and $\tilde{M}_{0}$ by identifying

$$
\{1\} \times \bigsqcup_{\sigma \in K^{\text {top }}}\{\sigma\} \times \operatorname{Int} \Delta^{k}
$$

with $\partial \tilde{M}^{*}$. We conclude that

$$
\partial\left(\left.\tilde{F}\right|_{\tilde{M}}\right)=-\left.F\right|_{M}
$$

i.e. $\left.F\right|_{M}$ and $h$ represent the zero element in $\mathcal{H}_{k}(M)$. 


\section{REFERENCES}

[BM] M. Barrett and J. Milnor, An Example of Anomalous Singular Homology, Proc. AMS. 13, 1962, 293-297. MR0137110 (25:566)

[FO] K. Fukaya and K. Ono, Arnold Conjecture and Gromov-Witten Invariant, Topology 38 (1999), no. 5, 933-1048. MR1688434 (2000j:53116)

[G] M. Gromov, Pseudoholomorphic Curves in Symplectic Manifolds, Invent. Math. 82 (1985), no. 2, 307-347. MR809718 (87j:53053)

[K] P. Kahn, Pseudohomology and Homology, math.AT/0111223.

[LT] J. Li and G. Tian, Virtual Moduli Cycles and Gromov-Witten Invariants of General Symplectic Manifolds, Topics in Symplectic 4-Manifolds, 47-83, First Int. Press Lect. Ser., I, Internat. Press, 1998. MR1635695 (2000d:53137)

[McSa] D. McDuff and D. Salamon, Introduction to J-Holomorphic Curves, AMS, 1994.

[Mu1] J. Munkres, Topology, Prentice Hall, 1999.

[Mu2] J. Munkres, Elements of Algebraic Topology, Addison-Wesley, 1994. MR755006 (85m:55001)

[RT] Y. Ruan and G. Tian, A Mathematical Theory of Quantum Cohomology, J. Diff. Geom. 42 (1995), no. 2, 259-367. MR1366548 (96m:58033)

[Sc] M. Schwartz, Equivalences for Morse Homology, Geometry and Topology in Dynamics, 197-216, Contemp. Math. 246, AMS, 1999. MR1732382 (2000j:57070)

[Z1] A. Zinger, Counting Rational Curves of Arbitrary Shape in Projective Spaces, Geom. Top. 9 (2005), 571-697. MR2140990 (2006e:14076)

[Z2] A. Zinger, Reduced Genus-One Gromov-Witten Invariants, math.SG/0507103.

Department of Mathematics, SUNY, Stony Brook, New York 11790-3651

E-mail address: azinger@math.sunysb.edu 